\title{
Microviscosity, microdiffusivity, and normal stresses in colloidal dispersions
}

\author{
R. N. Zia ${ }^{\text {a) }}$ \\ Department of Mechanical Engineering, California Institute of Technology, \\ Pasadena, CA 91125
}

J. F. Brady

Department of Mechanical Engineering and Department of Chemical Engineering,
California Institute of Technology, Pasadena, CA 91125

(Received 25 October 2011; final revision received 10 May 2012;

published 20 June 2012)

\begin{abstract}
Synopsis
In active, nonlinear microrheology, a Brownian "probe" particle is driven through a complex fluid and its motion tracked in order to infer the mechanical properties of the embedding material. In the absence of external forcing, the probe and background particles form an equilibrium microstructure that fluctuates thermally. Probe motion through the medium distorts the microstructure; the character of this deformation, and hence its influence on probe motion, depends on the strength with which the probe is forced, $F^{\mathrm{ext}}$, compared to thermal forces, $k T / b$, defining a Péclet number, $P e=F^{\mathrm{ext}} /(k T / b)$, where $k T$ is the thermal energy and $b$ is the characteristic microstructural length scale. Recent studies showed that the mean probe speed can be interpreted as the effective material viscosity, whereas fluctuations in probe velocity give rise to an anisotropic force-induced diffusive spread of its trajectory. The viscosity and diffusivity can thus be obtained by two simple quantities - mean and mean-square displacement of the probe. The notion that diffusive flux is driven by stress gradients leads to the idea that the stress can be related directly to the microdiffusivity, and thus the anisotropy of the diffusion tensor reflects the presence of normal stress differences in nonlinear microrheology. In this study, a connection is made between diffusion and stress gradients, and a relation between the particle-phase stress and the diffusivity and viscosity is derived for a probe particle moving through a colloidal dispersion. This relation is shown to agree with two standard micromechanical definitions of the stress, suggesting that the normal stresses and normal stress differences can be measured in nonlinear microrheological experiments if both the mean and mean-square motion of the probe are monitored. Owing to the axisymmetry of the motion about a spherical probe, the second normal stress difference is zero, while the first normal stress difference is linear in $P e$ for $P e \gg 1$ and vanishes as $P e^{4}$ for $P e \ll 1$. The expression obtained for stress-induced migration can be viewed as a generalized nonequilibrium Stokes-Einstein relation. A final connection is made between the stress and an "effective temperature" of the medium, prompting the interpretation of the particle stress as the energy density, and the expression for osmotic pressure as a "nonequilibrium equation of state.” (C) 2012 The Society of Rheology. [http://dx.doi.org/10.1122/1.4722880]
\end{abstract}

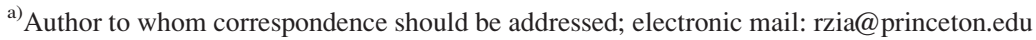




\section{INTRODUCTION}

Complex fluids encompass systems as diverse as toothpaste, the interior of the cell, and sprayable solar panels, to name just a few. Uniting these materials is their rich multiphase structure: a collection of microscopic domains or particles which forms a microstructure that is embedded in a continuum material. Imposing a flow in the material deforms this microstructure, which can give rise to dramatic changes in flow behavior and material properties. An understanding of the rheological properties of complex media is critical to their processing, dispensing, durability, and dynamic performance. Traditional rheology experiments are carried out by imposing a bulk shearing motion on a sample of material in a viscometer. The shearing motion may be constant, or an oscillatory shear may be applied in order to explore time-dependent phenomena such as viscoelasticity. Barnes et al. (1989) provide a thorough review of traditional rheological techniques. Theoretical rheology has the additional goal of deriving constitutive relations between stress and strain rate from statistical mechanics at the particle microstructural level. But recent years have seen a dramatic growth in demand for exploring microscale systems whose dynamic response properties must be measured at a much smaller length scale than is possible to probe with conventional viscometers. Understanding spatial variation in network strength in hydrogels, interrogating rare biological fluids, and determination of the diffusive speed of nanotherapeutic devices inside the cell are a just few examples [Lukacs et al. (1999); Verkman (2002); Suh et al. (2003); Heath et al. (2009); Olsen et al. (2010)]. The fundamental process underlying each of these problems is material response to dynamic forcing by a microscale probe- a microscopic version of rheometry. This type of microscale probing, known as "microrheology," has a long history, yet has re-emerged in the last decade as a powerful technique for material evaluation.

Microrheology comprises a theoretical framework and experimental technique in which the motion of a Brownian particle (or set of particles) is tracked in order to determine the properties of the surrounding medium [MacKintosh and Schmidt (1999)], the origins of which can be traced to the work of Einstein (1906) and Perrin (1909). Due to the small length scales intrinsic to colloidal dispersions, microrheology has become more practicable in the last two decades [Crocker and Grier (1996)]. Much work has since followed, including the extension of the Stokes-Einstein relation to viscoelastic materials [Mason and Weitz (1995)], and studies of the effect of probe size [Lu and Solomon (2002)] and shape [Khair and Brady (2008)]. Microrheological techniques have been used to study a diverse set of systems: cells [Bausch et al. (1998); Guilford et al. (1999); Lau et al. (2003)], actin networks [Gisler and Weitz (1999); Ziemann et al. (1994)], gelatin [Freundlich and Seifriz (1923)], deoxyribose nucleic acid (DNA) and polyethylene oxide solutions [Mason et al. (2005)], and the behavior of colloids near the glass transition [Habdas et al. (2004)], as well as fundamental interactions between pairs of colloidal spheres [Crocker (1997); Crocker et al. (2000); Levine and Lubensky (2000)] and entropic forces in binary colloids [Crocker et al. (1999)]. Microrheology has also been proposed as a tool for studying basic physics in atomic or molecular systems and for high-throughput material screening [Breedveld and Pine (2003); Schultz and Furst (2011)].

Both the equilibrium (linear response) properties and the nonlinear response of the material can be probed, via passive and active microrheology, respectively. In the former, thermal fluctuations of a particle cause it to undergo a random-walk process; equilibrium and linear viscoelastic properties are then obtained by correlating the random thermally driven displacements of tracers to the complex modulus through a generalized StokesEinstein relation. In order to obtain nonlinear-response properties, however, the material 
must be driven out of equilibrium. In this active (or nonlinear) microrheology regime, tracer particles undergo displacements due not only to random thermal fluctuations but also due to the application of an external force applied directly to the tracer, or "probe," or by applying a constant or oscillatory external force to the particles, for example, or by using optical tweezers or magnetic fields [Furst (2005); Meyer et al. (2005); Habdas et al. (2004); Wilson et al. (2009); Sriram et al. (2009)]. As with macrorheology, dynamic response properties such as viscosity can then be measured. Since the tracer interrogates the material at the microscopic length scale, much smaller samples are required compared to traditional macrorheology and localized material heterogeneity can be explored; microrheology thus holds a particular benefit for rare biological materials and small systems such as cells. The theory that predicts the microviscosity and microdiffusivity of dilute systems of colloids - and defines the relationship between microrheology and macrorheology — has recently been established [Squires and Brady (2005); Khair and Brady (2005, 2006); Zia and Brady (2010); Zia (2011)]. Recent experiments confirm the theory and raise important questions and concerns [Meyer et al. (2005); Squires (2008); Wilson et al. (2009)] which are discussed below.

One of the primary objectives of traditional macroscale rheology is to understand and characterize the relationship between deformation rate and bulk material stress, e.g., the shear viscosity. In the present investigation, material behavior is studied through the framework of nonlinear microrheology where the Brownian probe particle is actively driven through a complex fluid and its motion connected to the mechanical properties of the embedding material. Squires and Brady (2005) and Khair and Brady (2006) showed that the effective viscosity in dilute dispersions can be determined by relating the external force to mean probe velocity via application of Stokes' drag law. Zia and Brady (2010) showed further that the collisions between the probe and the embedded particles cause the probe to undergo a random-walk process; this force-induced diffusion or "microdiffusivity" can be determined by monitoring the fluctuating (mean-square) motion of the probe. But no corresponding theory has yet been put forth to determine the full material stress via microrheology. Due to the axisymmetric geometry surrounding the probe, recent approaches in nonlinear microrheology were able to produce only a scalar evaluation of the stress. But it has been proposed in previous studies of complex fluids that diffusion is driven by stress gradients [Einstein (1906); Batchelor (1976); Brady and Morris (1997)]. If such a connection can be made in nonlinear microrheology, then by simply monitoring the mean and mean-square motion of a probe, one would obtain a full rheological picture of a material: viscosity, diffusivity, and stress. Our recent work [Zia and Brady (2010)] showed that the microdiffusivity tensor is transversely anisotropic, suggesting that a connection between stress and diffusivity would provide a measure of normal stress differences.

Previous observations that stress gradients drive diffusion focus mainly on equilibrium colloidal suspensions. Einstein (1906) proposed this idea in his model of the Brownian motion. He asserted that gradients in the osmotic pressure drive diffusion. In a slightly different approach, Batchelor (1976) showed that gradients in the chemical potential are the driving force for diffusive flux in single- and multispecies (near) equilibrium colloidal dispersions. Connecting the two approaches yields the simple prediction that gradients in the stress in colloidal dispersions drive diffusion: $\partial \boldsymbol{\Sigma} / \partial n \propto \boldsymbol{D}$, where $\boldsymbol{\Sigma}$ is the stress, $\boldsymbol{D}$ is the diffusion tensor, and $n$ is the number density of particles. If this relation holds true for nonequilibrium suspensions, then simply watching the motion of a single particle would yield an extraordinary amount of information. It is the primary goal of this investigation to develop such an expression and, from it, determine a relationship between stress gradients and particle migration and diffusion. 
The remainder of this paper is organized as follows: A brief review of gradient-driven diffusion in equilibrium systems is given in Sec. II. The connection between the osmotic pressure and the chemical potential shows that diffusion also arises due to osmotic pressure gradients. This suggests a more general relation between stress gradients and diffusion in nonequilibrium systems: $\boldsymbol{D}=-\boldsymbol{M} \cdot(\nabla \cdot \boldsymbol{\Sigma})$, where $\boldsymbol{M}$ is the appropriate hydrodynamic mobility tensor. The validity of this relation in nonequilibrium systems is examined in Sec. II A using the test case of microrheology of a dilute colloidal dispersion. The results show fair agreement with results obtained via a detailed micromechanical approach and Brownian dynamics simulation, but quantitative differences indicate that the proposed equilibrium relation does not fully account for particle migration. The primary difference between systems for which the relation holds and our nonlinear microrheology system is that the former are at equilibrium-which can be described by an equation of state-while the latter is not at equilibrium — and must be described by an equation of motion.

A momentum balance approach is proposed in Sec. III, in which the divergence of the stress is balanced by body forces. The constitutive form for the forces in Stokes' flow reveals that stress gradients do not simply drive diffusion but also drive mean particle flux. Familiar expressions for particle flux in colloids make it evident that one must consider not only diffusive flux but also advective flux. The nonlinear microrheology test case is carefully revisited; because particles migrate due to diffusion and also due to being advected by the mean flow, the model suggests that a general relation must contain both advective and diffusive contributions. The resulting relationship between stress, diffusion, and viscous drag confirms that stress gradients drive diffusive and advective fluxes in nonequilibrium systems, and it is proposed that this can be viewed as a nonequilibrium Stokes-Einstein relation.

The normal stress, normal stress differences, and the osmotic pressure are obtained analytically for small and large $P e$, and numerically for the full range of $P e$. The results are given in Sec. IV, where they are compared to the stress derived via a traditional micromechanical (Smoluchowski) approach (Sec. IV A) and then in Sec. IV B to measurements obtained via Brownian dynamics simulation. In Sec. V, the proposed theory is compared to macrorheology for the stress in a dilute dispersion of colloids undergoing simple shear. An important question raised in the literature is whether the nonviscometric nature of the flow around the probe can predict bulk material properties. This question is addressed in Sec. VI. In Sec. VII, it is proposed that the nonequilibrium particle stress is a measure of the ratio of fluctuation to dissipation, which suggests that our final result represents a nonequilibrium fluctuation-dissipation relation, and the expression for the osmotic pressure a "nonequilibrium equation of state." Section VIII gives a guideline for application of the theory to experimental practice. The study is concluded in Sec. IX with a discussion, including connection of the theory to general complex media.

\section{STRESS-INDUCED DIFFUSION-A FIRST LOOK}

In his theory of Brownian motion, Einstein (1906) presented two approaches to connect thermal motion and diffusion to mechanical motion and hydrodynamics. A central assumption in both approaches is thermodynamic equilibrium between the solvent and the suspended particles. In one approach, a dilute suspension of colloids is imagined to be settling in a container under gravity thereby generating a weak spatial concentration gradient from bottom to top. The gradient in the colloid number density $n$ drives a diffusive flux upward. Because the system is at equilibrium in the external gravitational 
potential $V_{g}$, the diffusive flux, $\boldsymbol{j}_{D}$, is exactly balanced by the advective flux owing to sedimentation, $\boldsymbol{j}_{\text {sed }}$. Assuming a Fickian diffusion process, $\boldsymbol{j}_{D}=-\boldsymbol{D} \cdot \nabla n$, and an advective flux given by hydrodynamic mobility, $\boldsymbol{M}$, times the driving force of gravity, $\boldsymbol{j}_{\text {sed }}=-n \boldsymbol{M} \cdot \nabla V_{g}$, and recognizing that the equilibrium Boltzmann distribution, $n \sim \exp \left(-V_{g} / k T\right)$, applies, Einstein deduced that

$$
\boldsymbol{D}=k T M,
$$

relating the translational diffusivity $\boldsymbol{D}$ to the thermal energy $k T$ and the hydrodynamic mobility $\boldsymbol{M}$. For a spherical particle of radius $a$, the Stokes mobility $\boldsymbol{M}=\boldsymbol{I} / 6 \pi \eta a$, giving $\boldsymbol{D}=k T /(6 \pi \eta a) \boldsymbol{I}$, where $\eta$ is the viscosity of the solvent and $\boldsymbol{I}$ is the isotropic tensor.

In his second derivation, Einstein proposed that the driving force for diffusion is the gradient in the osmotic pressure, $\Pi$, of the particles. Recall that the osmotic pressure is the particles' contribution to the total pressure in the solvent-particle mixture. For dilute colloids, $\Pi=n k T$, and Eq. (1) is recovered.

More modern irreversible thermodynamics [see, e.g., DeGroot and Mazur (1984)] and statistical hydrodynamical treatments [Batchelor (1976)] show that diffusion is driven by gradients in the chemical potential $\mu$ of the particles resulting in

$$
\boldsymbol{D}=\boldsymbol{M} n \frac{\partial \mu}{\partial n}
$$

Thermodynamics relates the chemical potential to the osmotic pressure of the dilute colloids, whence

$$
\boldsymbol{D}=\boldsymbol{M} \frac{\partial \Pi}{\partial n}
$$

recovering Einstein's second argument.

But, whereas the chemical potential is only defined at equilibrium, the osmotic pressure has a purely mechanical definition that applies equally well away from equilibrium [Brady (1993)]. Recalling that mechanically the pressure is minus one-third the trace of the stress tensor, we are prompted to propose a more general form for the diffusivity,

$$
\boldsymbol{D}=\boldsymbol{M} \cdot \frac{\partial \boldsymbol{\Sigma}}{\partial n}
$$

where $\Sigma$ is the particles' contribution to the total stress in the suspension-the particlephase stress tensor. If such a relation holds out of equilibrium, a rather simple measurement - the diffusivity - would yield information about the nonequilibrium stress state of the material. We shall now test this simple idea through the framework of nonequilibrium microrheology of a colloidal dispersion.

Our interest is in the nonequilibrium and nonlinear behavior when, for example, normal stress differences appear. In such a far-from equilibrium situation, the active deformation of the material's microstructure caused by the external forcing generates the nonequilibrium stress response and is also the source for the fluctuations experienced by a probe particle as it moves by advection (and diffusion) through the material, thus providing the link expressed by Eq. (4) between diffusion and stress.

In active nonlinear microrheology of colloidal dispersions, a probe particle of size $a$ is driven by an external force $\boldsymbol{F}^{\text {ext }}$ through an otherwise quiescent suspension of bath particles of size $b$ (a review of the microrheology model system can be found in 
Appendix A). We take both the probe and bath particles to behave as hard spheres, and in this first look neglect hydrodynamic interactions between probe and bath particles. This problem has recently received considerable attention in the literature and so its behavior is relatively well understood. As the probe is forced through the bath it distorts the structure of the surrounding bath particles and the deformed microstructure exerts an entropic reactive force on the probe, slowing its motion. The effective viscosity $\eta^{\text {eff }}$ is then defined from Stokes' drag law relating the external force to the average velocity of the probe [Squires and Brady (2005)]:

$$
\boldsymbol{F}^{\mathrm{ext}}=6 \pi \eta^{\mathrm{eff}} a\langle\boldsymbol{U}\rangle
$$

Here, $\langle\cdot\rangle$ is an ensemble average over a large number of noninteracting, i.e., very dilute probe particles moving through the bath, as is typically measured experimentally. A brief discussion of the theoretical description of the microviscosity can be found in Appendix A.

At any given instant, the probe's velocity is equal to its mean velocity plus a fluctuational component, $\boldsymbol{U}=\langle\boldsymbol{U}\rangle+\boldsymbol{U}^{\prime}$, as it encounters individual bath particles (i.e., microstructural fluctuations), while it is forced through the bath. These fluctuations give rise to a diffusive spread about the probe's mean motion. This force-induced microdiffusivity, $\boldsymbol{D}^{\text {micro }}$, was studied by Zia and Brady (2010) who showed that the microdiffusivity is anisotropic-motion along the direction of forcing exceeds that transverse to it. This microdiffusivity is also given by an ensemble average over the trajectories of many probes and can be viewed either as the self-diffusion of a probe particle or the down-gradient Fickian tracer diffusivity resulting from a weak concentration gradient of probes $\nabla n_{a}$ in a bath of background particles, where $n_{a}$ is the dilute probe concentration and the bath particles have concentration $n_{b}$ [see Rallison and Hinch (1986) for a thorough discussion of the connection between self-diffusion, gradient diffusion, and tracer diffusion].

For the "osmotic pressure" of the probes, we suppose a dilute concentration of probe particles with number density $n_{a}$ in a suspension of bath particles at number density $n_{b}\left(n_{a} \ll n_{b}\right)$. For hard-sphere particles, the probe particles' contribution to the suspension stress is given by [McQuarrie (1976); Brady (1993)]

$$
\langle\boldsymbol{\Sigma}\rangle=-n_{a} k T-\frac{1}{2} n_{a} n_{b} k T(a+b) \int_{r=a+b} \boldsymbol{n n} g(\boldsymbol{r}) d S,
$$

where $\boldsymbol{n}$ is the normal to the contact surface at probe-bath particle separation $r=a+b$, and $g(\boldsymbol{r})$ is the probe-bath pair-distribution function. Equation (6) is an exact formula (for hard spheres) for the stress contribution from the probe particles no matter what the concentration of bath (or probe) particles and for any type of forcing, e.g., shearing motion or an external force for microrheology. The stress (6) also applies at equilibrium but since our interest is in the nonequilibrium behavior we focus here on the departure from equilibrium,

$$
\left\langle\Sigma^{\mathrm{neq}}\right\rangle=-\frac{1}{2} n_{a} n_{b} k T(a+b) \int_{r=a+b} \boldsymbol{n n} g^{\mathrm{neq}}(\boldsymbol{r}) d S,
$$

where $\Sigma^{\text {neq }}$ and $g^{\text {neq }}(\boldsymbol{r})$ are the nonequilibrium stress and probe-bath pair-distribution function, respectively, and $\boldsymbol{r}$ is the separation between a pair of particles. The departure from equilibrium of the microstructure, $g^{\text {neq }}(\boldsymbol{r})=g(\boldsymbol{r})-g^{o}(\boldsymbol{r})$, where $g^{o}$ is the equilibrium configuration, is caused by the external forcing of the probe and is precisely what determines the entropic reactive force and microviscosity (see Appendix A). 
To make the connection between the stress and diffusion, we note that $\partial\left\langle\Sigma^{\text {neq }}\right\rangle / \partial n_{a}$ $=\left\langle\Sigma^{\text {neq }}\right\rangle / n_{a}$ as must be true for infinitely dilute $n_{a}$ as $\left\langle\Sigma^{\text {neq }}\right\rangle$ is extensive of degree one in $n_{a}$, and both $\Sigma^{\text {neq }}$ and $\boldsymbol{D}^{\text {micro }}$ are proportional to the concentration of bath particles $n_{b}$. Thus, for a dilute bath $\phi_{b}=4 \pi b^{3} n_{b} / 3 \ll 1$, we can use the isolated probe mobility $\boldsymbol{M}=\boldsymbol{I} / 6 \pi \eta a$ in Eq. (4) to write

$$
-\frac{\left\langle\Sigma^{\text {neq }}\right\rangle}{n_{a} k T}=\frac{D^{\text {micro }}}{D_{a}},
$$

where $D_{a}=k T / 6 \pi \eta a$ is the diffusion coefficient of the probe alone in the solvent.

\section{A. Normal stresses, normal stress differences, and the osmotic pressure}

In Fig. 1, we plot the microdiffusivity - the right-hand side of Eq. (8) - as determined by Zia and Brady (2010) along with the stress — the left-hand side of Eq. (8) — determined from Eq. (7), as a function of strength of the external forcing, the Péclet number $P e=F^{\mathrm{ext}} b / k T$. Components parallel (longitudinal) and perpendicular (transverse) to the direction of forcing are shown in Figs. 1(a) and 1(b), respectively. For simplicity, we shall take the probe-to-bath particle size ratio $a / b$ to be unity, but this need not be the case, as is discussed in Sec IX. The qualitative agreement over the entire range of $P e$ is good. At small $P e$, the stress and diffusivity show the expected $P e^{2}$ scaling-the probe samples the $O(P e)$ velocity fluctuations diffusively, i.e., Taylor dispersion, while at high $P e$ both are proportional to $P e$, reflecting the mechanical (advective) nature of the deformation and scattering of the probe by the bath particles.

The good qualitative agreement between the stress and diffusion strongly supports the hypothesis that the same fundamental mechanisms responsible for the force-induced diffusion also govern the stress response of the material, and it offers a simple means to determine normal stresses in a material: watch a particle diffuse. However, there are quantitative differences: at low $P e$ the diffusivity is larger than the longitudinal stress, while at high $P e$ it is less than the transverse stress. These quantitative differences suggest that the relation (8) may be incomplete. Furthermore, the deformation of the material caused by the moving probe is axisymmetric about the probe and has the character of an

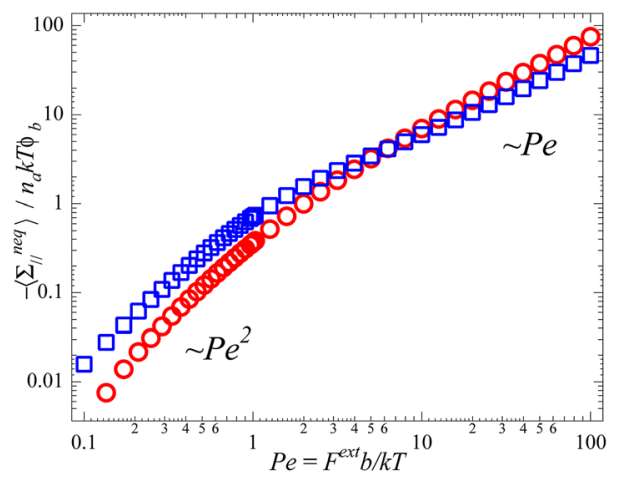

(a) Longitudinal normal stress

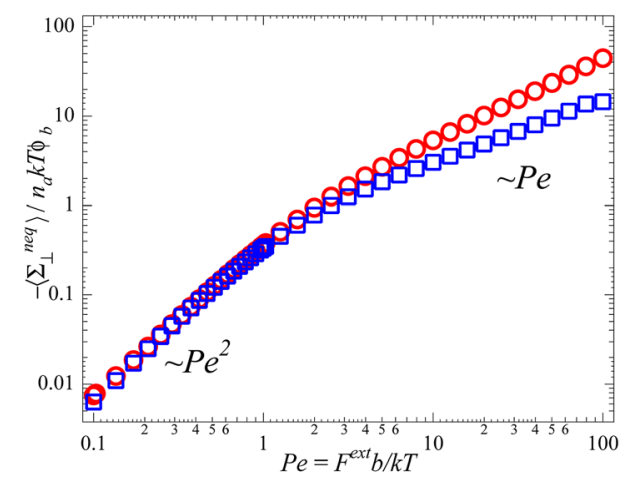

(b) Transverse normal stress

FIG. 1. Normal stress scaled by $n_{a} k T$ and volume fraction of bath particles $\phi_{b}$ for size ratio $a / b=1$. Red circles represent the micromechanically derived stress-the left-hand side of Eq. (8) via Eq. (7): (O) $(3 / \pi) \int \boldsymbol{n} \boldsymbol{n} g^{\text {neq }}(\boldsymbol{r}) d \Omega$. Blue squares represent the right-hand side of Eq. (8) as determined by Zia and Brady (2010): ( $\square$ ) $\boldsymbol{D}^{\text {micro }} / D_{a} \phi_{b}$. Longitudinal components of both quantities shown in (a) and transverse components shown in (b). 
elongational rather than a shear flow. In a simple shear flow, the off-diagonal components of the stress tensor, $\Sigma_{x y}$, say, are related to the viscosity, while the diagonal components give the normal stresses. In an elongational flow, however, all components of the stress tensor lie along the diagonal and therefore are "normal" even though in a simple Newtonian fluid undergoing such a flow, normal stresses are purely viscous. Thus, we should expect viscous stresses - the microviscosity - to enter into the relation (8). The relation between diffusion and stress thus requires a closer look.

\section{STRESS-INDUCED MIGRATION-A CLOSER LOOK}

The approach we shall take here is to consider a material through which there are a large number of probe particles moving in response to an external force. We then write a momentum balance on the probes viewed as a phase. The importance of fluid inertia relative to the viscous forces is characterized by the Reynolds number, $R e=\rho U a / \eta$, where $U$ is the characteristic velocity of a moving probe, and for micron-sized particles $R e \ll 1$ so that the fluid mechanics are governed by Stokes flow and the inertial term in the momentum balance can be neglected. The momentum balance has the usual form which for small Reynolds numbers reads

$$
0=\nabla \cdot\langle\boldsymbol{\Sigma}\rangle+n_{a}\left\langle\boldsymbol{F}^{\mathrm{ext}}\right\rangle+n_{a}\left\langle\boldsymbol{F}^{\mathrm{int}}\right\rangle
$$

where the angle brackets $\langle\cdot\rangle$ indicate a volume or ensemble average. In Eq. (9), $\Sigma$ is the stress tensor associated with the probe phase, $n_{a}$ is the number density of probes, $\boldsymbol{F}^{\mathrm{ext}}$ is the external force exerted on the probe particles, and $\boldsymbol{F}^{\text {int }}$ is the interactive force between the probes and the surrounding material. The probes are taken to be so dilute that they do not interact with one other. The momentum balance (9) follows from volume averaging of the pointwise Cauchy equation of motion that applies at each point in the material [see, e.g., Batchelor (1977)].

A part of the interactive force $\boldsymbol{F}^{\text {int }}$ between the probes and the surrounding medium is caused by their relative motion; thus, the interactive force can be written constitutively as

$$
n_{a}\left\langle\boldsymbol{F}^{\text {int }}\right\rangle=-n_{a}\langle\boldsymbol{R} \cdot \boldsymbol{U}\rangle+\left\langle\boldsymbol{f}_{P}\right\rangle=-\langle\boldsymbol{R} \cdot \boldsymbol{j}\rangle+\left\langle\boldsymbol{f}_{P}\right\rangle,
$$

where $\boldsymbol{R}$ is the tensor describing the resistance of the medium to the probe's motion, $\boldsymbol{j}=n_{a} \boldsymbol{U}$ is the probe flux, and we have presumed that the overall medium is not moving, for otherwise one would have the probe velocity relative to the medium. The other part of the interaction force is denoted $f_{P}$ and arises from the restriction of volume accessible to the probes by the bath particles, as discussed below. Thus, the probe-phase momentum balance can be written as

$$
0=\nabla \cdot\langle\boldsymbol{\Sigma}\rangle+n_{a} \boldsymbol{F}^{\mathrm{ext}}-\langle\boldsymbol{R} \cdot \boldsymbol{j}\rangle+\left\langle\boldsymbol{f}_{P}\right\rangle .
$$

Our goal is to use Eq. (11) to relate the nonequilibrium stress $\left\langle\Sigma^{\text {neq }}\right\rangle$ to the force-induced diffusivity as in Eqs. (4) and (8).

We first note that Eq. (11) should recover the diffusion of the probe in the absence of any external forcing - the diffusion problem considered by Einstein. The weak perturbation to the distribution of bath particles in this case is equivalent to the forcing due to thermal fluctuations, following linear-response theory. We denote this problem as

$$
0=\nabla \cdot\left\langle\Sigma^{o}\right\rangle-\left\langle\boldsymbol{R}^{o} \cdot \boldsymbol{j}^{o}\right\rangle+\left\langle\boldsymbol{f}_{P}^{o}\right\rangle,
$$


with $\boldsymbol{j}^{o}=-\boldsymbol{D}^{o} \cdot \nabla n_{a}$. At infinite dilution we recover Einstein's second perspective on diffusion, $\left\langle\Sigma^{o}\right\rangle=-n_{a} k T \boldsymbol{I}$ (with $\boldsymbol{R}^{o} \cdot \boldsymbol{D}^{o}=k T \boldsymbol{I}$ ). Next, for a dilute suspension of interacting particles $\left\langle\Sigma^{o}\right\rangle=-n_{a} k T\left(1+4 \phi_{b}\right) \boldsymbol{I}$ (with $\left.\boldsymbol{R}^{o} \cdot \boldsymbol{D}^{o}=k T\left(1-2 \phi_{b}\right) \boldsymbol{I}\right)$. Here, the $O\left(\phi_{b}\right)$ contribution to the stress corresponds to the interactive force density, or pressure, $f_{P}$, which acts on the probes due to the restriction of volume available to the probes by the bath particles.

In addition to pertaining to diffusive motion, Eq. (11) applies to the average motion of the probes owing to an external forcing when there are no gradients (no fluctuations) present. Imagine the probes moving due to an external potential; the average fall speed would give an advective flux $\boldsymbol{j}^{\mathrm{adv}}$ in the force balance

$$
0=n_{a}\left\langle\boldsymbol{F}^{\mathrm{ext}}\right\rangle-\langle\boldsymbol{R} \cdot \boldsymbol{j}\rangle^{\mathrm{adv}} .
$$

The flux of probes $\boldsymbol{j}^{\text {adv }}=n_{a} \boldsymbol{U}$ is reduced from the Stokes velocity due to the hindrance of the bath particles, as discussed in Sec. II.

Finally, Eq. (11) applies to the more general case of probes moving with both a mean and fluctuating motion,

$$
\boldsymbol{j}=\boldsymbol{j}^{\mathrm{adv}}+\boldsymbol{j}^{\mathrm{diff}} .
$$

Imagine here that the probes are falling due to gravity, which allows their velocities to fluctuate. The interactive force thus has both mean and fluctuating components, and the full nonequilibrium momentum balance, including the equilibrium diffusive behavior, becomes

$$
0=\nabla \cdot\left\langle\boldsymbol{\Sigma}^{o}\right\rangle+\nabla \cdot\left\langle\boldsymbol{\Sigma}^{\mathrm{neq}}\right\rangle-\left\langle\boldsymbol{R}^{o} \cdot \boldsymbol{j}^{o}\right\rangle-\left\langle(\boldsymbol{R} \cdot \boldsymbol{j})^{\mathrm{neq}}\right\rangle+\left\langle\boldsymbol{f}_{P}^{0}\right\rangle+\left\langle\boldsymbol{f}_{P}^{\mathrm{neq}}\right\rangle+n_{a}\left\langle\boldsymbol{F}^{\mathrm{ext}}\right\rangle,
$$

where the term $\left\langle(\boldsymbol{R} \cdot \boldsymbol{j})^{\text {neq }}\right\rangle$ corresponds to flow-induced flux, and in the present problem includes both advective and diffusive contributions. By definition, the average of a product is the product of the averages plus the average of the fluctuations: $\left\langle(\boldsymbol{R} \cdot \boldsymbol{j})^{\text {neq }}\right\rangle=\left\langle\boldsymbol{R}^{\text {neq }}\right\rangle \cdot\left\langle\boldsymbol{j}^{\text {neq }}\right\rangle+\left\langle\boldsymbol{R}^{\text {neq }} \cdot \boldsymbol{j}^{\text {neq }}\right\rangle$. The first term on the right-hand side of this expression corresponds to the mean advective motion, $\boldsymbol{j}^{\text {adv }}$, and the effective viscosity. The second term corresponds to fluctuating probe motion and the diffusive flux $\boldsymbol{j}^{\text {diff }}$. A Fickian model for the diffusive flux gives $\boldsymbol{j}^{\text {diff }}=-\boldsymbol{D}^{\text {micro }} \cdot \nabla n_{a}$, where $\boldsymbol{D}^{\text {micro }}$ is the $O\left(\phi_{b}\right)$ force-induced diffusion of the probes. Combining Eqs. (12), (14), and (15), we have

$$
-\nabla \cdot\left\langle\boldsymbol{\Sigma}^{\text {neq }}\right\rangle=\boldsymbol{R} \cdot \boldsymbol{D}^{\text {micro }} \cdot \nabla n_{a}-\langle\boldsymbol{R} \cdot \boldsymbol{j}\rangle^{\text {adv }}+\left\langle\boldsymbol{f}_{P}^{\text {neq }}\right\rangle+n_{a}\left\langle\boldsymbol{F}^{\text {ext }}\right\rangle .
$$

Noting that

$$
\nabla \cdot\left\langle\Sigma^{\mathrm{neq}}\right\rangle=\frac{\partial\left\langle\Sigma^{\mathrm{neq}}\right\rangle}{\partial n_{a}} \cdot \nabla n_{a}=\frac{\left\langle\Sigma^{\mathrm{neq}}\right\rangle}{n_{a}} \cdot \nabla n_{a}
$$

and for the dilute bath microrheology problem,

$$
\boldsymbol{R}=6 \pi \eta a \boldsymbol{I}+O\left(\phi_{b}\right),
$$

we see that the first-look expression (8) is given by only the first term on the right-hand side of Eq. (16). The additional advective components of the flux in Eq. (16) contribute to the relation between nonequilibrium stress and force-induced diffusion. 
The coarse-grained, averaged level of the macroscopic probe-phase momentum balance is not a detailed micromechanical model and thus the additional terms on the righthand side of Eq. (16) need to be constitutively modeled. We begin with the advective term $\langle\boldsymbol{R} \cdot \boldsymbol{j}\rangle^{\text {adv }}$. For a purely advective motion balancing the external forcing [Eq. (13)], we determined that the strength of the advective flux is linked to the effective viscosity of the medium,

$$
\left\langle\boldsymbol{F}^{\mathrm{ext}}\right\rangle_{U}=\frac{\eta_{U}^{\mathrm{eff}}}{\eta} \boldsymbol{R} \cdot\langle\boldsymbol{U}\rangle
$$

Here, we have suppressed any probe gradients, which amounts to suppressing any fluctuational motion of the probe. The subscript $U$ denotes that the probe velocity is held constant. Such motion corresponds to one of a constant-velocity probe rather than of constant force; at constant velocity, the resistance to probe motion is larger than at constant force because the probe must plow bath particles out of its way without any change in its speed. In the constant-force mode, where the probe's velocity can fluctuate, the resistance to probe motion is not as great because the probe can adjust its speed when encountering regions of relatively higher (or lower) volume fraction, and move around them. Hence, the hydrodynamic mobility of the fluctuating probe is higher than that of the constant-velocity probe. The effective viscosity in the constant external-force case is denoted with a subscript $F$,

$$
\left\langle\boldsymbol{F}^{\mathrm{ext}}\right\rangle_{F}=\frac{\eta_{F}^{\mathrm{eff}}}{\eta} \boldsymbol{R} \cdot\langle\boldsymbol{U}\rangle
$$

where we are careful to note that $\eta_{U}^{\text {eff }} \neq \eta_{F}^{\text {eff }}$. The effective viscosity may then be written as

$$
\frac{\eta^{\mathrm{eff}}}{\eta} \approx 1+\frac{\eta^{\mathrm{micro}}}{\eta},
$$

where the microviscosity $\eta^{\text {micro }} / \eta$ is the viscous drag of the particle microstructureabove and beyond the solvent viscosity (cf. Appendix A). In general, it includes contributions due to hydrodynamic, interparticle, and Brownian drag: $\eta^{\text {micro }}=\eta^{\text {micro, } H}$ $+\eta^{\text {micro, } P}+\eta^{\text {micro, } B}$. In the present case only the interparticle contribution is present, and in the dilute limit, $\eta^{\text {micro }} / \eta \sim O\left(\phi_{b}\right)$.

The advective interactive force $\langle\boldsymbol{R} \cdot \boldsymbol{j}\rangle^{\mathrm{adv}}$ acting on the probes must properly account for the ability of the probe to change its speed, i.e., it must account for the difference between constant-force and constant-velocity motion (discussed further in Appendix B). In the hydrodynamics resistance formalism, the former corresponds to a mobility problem, whereas the latter corresponds to a resistance problem, a difference sometimes underappreciated in its importance. In constant-force mode, the probe's ability to "wiggle around" the other particles results in an increase in probe mobility $\boldsymbol{M}_{F}=\boldsymbol{R}_{F}^{-1}$ compared to the fixed-velocity case: $\boldsymbol{R}_{U} \neq \boldsymbol{R}_{F}$. Let us denote the increased mobility (reduced resistance) in the constant-force case as $\left\langle\boldsymbol{M}_{F}^{-1}\right\rangle=\left\langle\boldsymbol{R}_{U}-\tilde{\boldsymbol{R}}\right\rangle$, where $\tilde{R}$ is the reduction in viscous drag, $\tilde{\eta}$, say, for a constant-force probe. In the present case, since the bath is dilute $\eta_{U}^{\text {micro }}=2 \eta_{F}^{\text {micro }}($ cf. Appendix B) and we write

$$
-\nabla \cdot\left\langle\boldsymbol{\Sigma}^{\mathrm{neq}}\right\rangle=\boldsymbol{R} \cdot \boldsymbol{D}^{\text {micro }} \cdot \nabla n_{a}-n_{a} \boldsymbol{R} \cdot\langle\boldsymbol{U}\rangle\left(\frac{\eta_{F}^{\text {micro }}}{\eta}-\frac{\tilde{\eta}}{\eta}\right)+\left\langle\boldsymbol{f}_{P}^{\mathrm{neq}}\right\rangle .
$$


The additional resistance, $\tilde{\eta}$, experienced by the constant-velocity probe is proportional to the number of particles that get in the probe's way, i.e., $\tilde{\eta} / \eta \sim O\left(\phi_{b}\right)$. In order for those particles to move out of the probe's way, they must each overcome solvent drag, and thus we propose the simple estimate $\tilde{\eta} /\left(\eta \phi_{b}\right)=1$. Inserting this into the momentum balance gives

$$
-\nabla \cdot\left\langle\Sigma^{\mathrm{neq}}\right\rangle=\boldsymbol{R} \cdot \boldsymbol{D}^{\mathrm{micro}} \cdot \nabla n_{a}-n_{a} \boldsymbol{R} \cdot\langle\boldsymbol{U}\rangle \phi_{b}\left(\frac{\eta_{F}^{\text {micro }}}{\eta \phi_{b}}-1\right)+\left\langle\boldsymbol{f}_{P}^{\mathrm{neq}}\right\rangle .
$$

We now turn our attention to the pressure term $\left\langle f_{P}\right\rangle$. Recall that diffusion-particle fluctuations-in equilibrium suspensions is associated with an isotropic osmotic pressure. These thermal particle fluctuations can be viewed in analogy to gas particles which collide with "walls" of a fictitious container that encloses them, giving rise to pressure. The colloidal particles also exert a pressure on fictitious walls- the osmotic pressure. At equilibrium, their thermal energy $k T$ determines how vigorously they fluctuate, and hence the osmotic pressure goes up and down with increasing or decreasing temperature. Above we found also that in the presence of bath particles, the osmotic pressure of the probe phase increases due to the restriction of accessible volume (reducing the "container" size) imposed by the presence of the bath particles. We can model these fluctuations as giving rise to an effective "temperature" for the probe's motion-a far-from equilibrium, forceinduced temperature. And such a temperature is proportional to the average of the probe velocity fluctuations squared, $\left\langle\boldsymbol{U}^{\prime} \boldsymbol{U}^{\prime}\right\rangle$, and thus is proportional to the force-induced diffusivity itself. Hence, we propose the model

$$
\left\langle\boldsymbol{f}_{P}\right\rangle \approx \boldsymbol{R} \frac{1}{3} \operatorname{tr}\left(\boldsymbol{D}^{\mathrm{micro}}\right) \cdot \nabla n_{a}
$$

where $\operatorname{tr}\left(\boldsymbol{D}^{\text {micro }}\right)$ is the trace of the force-induced microdiffusivity tensor. While there is no reason to suppose that that the force-induced temperature is isotropic, using the trace of $\boldsymbol{D}^{\text {micro }}$ makes this approximation. In essence, we are saying that the probe is much more "mobile" due to forcing than at equilibrium. Thus, the expression relating stress and probe flux becomes

$$
-\nabla \cdot\left\langle\boldsymbol{\Sigma}^{\mathrm{neq}}\right\rangle=\boldsymbol{R} \cdot \boldsymbol{D}^{\mathrm{micro}} \cdot \nabla n_{a}-n_{a} \boldsymbol{R} \cdot\langle\boldsymbol{U}\rangle \phi_{b}\left(\frac{\eta_{F}^{\text {micro }}}{\eta \phi_{b}}-1\right)+\boldsymbol{R} \cdot \frac{1}{3} \operatorname{tr}\left(\boldsymbol{D}^{\mathrm{micro}}\right) \boldsymbol{I} \cdot \nabla n_{a} .
$$

Recall from Eq. (23) that the constant-velocity behavior has been removed from the constant-force response; in a sense, the mean behavior has been removed and we are left only with the effects of fluctuation. All terms on the right-hand side of Eq. (25) thus have components proportional to the number-density gradient $\nabla n_{a}$. The steepness of this gradient can be approximated by a Fickian scaling,

$$
\nabla n_{a} \sim \frac{j}{D(P e)} \sim \frac{n_{a} U}{D(P e)} \sim \frac{n_{a} P e D_{a}}{D(P e) b},
$$

where $D(P e)$ is the coefficient of the force-induced diffusion which corresponds to $\boldsymbol{D}^{\text {micro }} / \phi_{b}$. Although on average the mean drag acts in the direction of forcing, its fluctuating components in the direction of the gradient are the same as those driving its diffusion [Zia and Brady (2010)]. Inserting this scaling into the momentum balance gives 


$$
-\frac{\Sigma^{\text {neq }}}{n_{a} k T}=\frac{\boldsymbol{D}^{\text {micro }}}{D_{a}}-\frac{\boldsymbol{D}^{\text {micro }}}{D_{a}} \cdot\left(\frac{\eta_{F}^{\text {micro }}}{\eta \phi_{b}}-1\right)+\frac{1}{3} \operatorname{tr}\left(\frac{\boldsymbol{D}^{\text {micro }}}{D_{a}}\right) \boldsymbol{I} .
$$

For a dilute bath, each of the terms on the right-hand side of Eq. (27) is linear in the volume fraction of bath particles $\phi_{b}$. The dimensionless stress is also independent of the number of probes, as must be true for infinitely dilute $n_{a}$ as $\left\langle\Sigma^{\text {neq }}\right\rangle$ is extensive of degree one in $n_{a}$. Regrouping terms gives the expression

$$
-\frac{\Sigma^{\mathrm{neq}}}{n_{a} k T}=\left(2-\frac{\eta^{\mathrm{micro}}}{\eta \phi_{b}}\right) \frac{\boldsymbol{D}^{\mathrm{micro}}}{D_{a}}+\frac{1}{3} \operatorname{tr}\left(\frac{\boldsymbol{D}^{\mathrm{micro}}}{D_{a}}\right) \boldsymbol{I} .
$$

Rearrangement leads to the Stokes-Einstein form,

$$
\boldsymbol{D}^{\mathrm{micro}}=-\left(\frac{\Sigma^{\mathrm{neq}}}{n_{a}}+\boldsymbol{P}\right) \cdot \boldsymbol{M}^{\mathrm{neq}}
$$

where, for compactness, we have replaced the isotropic pressure term with $\boldsymbol{P} \equiv \boldsymbol{R} \cdot \operatorname{tr}\left(\boldsymbol{D}^{\text {micro }}\right) \boldsymbol{I} / 3$. The nonequilibrium mobility tensor is given by

$$
\boldsymbol{M}^{\mathrm{neq}}=\left(2-\frac{\eta_{F}^{\mathrm{micro}}}{\eta \phi_{b}}\right)^{-1} \boldsymbol{M}^{o}
$$

and $\boldsymbol{M}^{o}=\boldsymbol{I} / 6 \pi \eta a$. Like the equilibrium Stokes-Einstein relation (1), the nonequilibrium expression (29) gives a relationship between fluctuation and dissipation. Fluctuations, $\boldsymbol{D}^{\text {micro }}$, driven by the energy, $\Sigma^{\text {neq }} / n_{a}$ and $\boldsymbol{P}$, are dissipated back to the solvent by viscous $\operatorname{drag}\left(\boldsymbol{M}^{\text {neq }}\right)^{-1}$.

\section{RESULTS}

An expression has been proposed that relates the stress to the particle flux in dilute colloidal dispersions, with a view toward its use with generalized complex media. The results from this approach are plotted and discussed below for the case of microrheology. The theory is compared to Brownian dynamics simulations in Sec. IV B and then compared to the stress tensor in dilute colloidal suspensions undergoing a bulk shearing motion in Sec. V.

\section{A. Normal stresses, normal stress differences, and the osmotic pressure}

In order to test the new expression, the right-hand side of Eq. (28) is plotted in Fig. 2 along with the left-hand side as computed via Eq. (7). Compared with the "first look" expression (8) shown in Fig. 1, agreement is now very good over the entire range of Péclet number. Accounting for the advective flux in the model yields qualitative and quantitative agreement between the coarse-grained and micromechanical approaches.

Normal stress differences can be important in the context of single-particle forcing in many physical systems; they may cause soft particles, e.g., subcellular organelles, to elongate, or even rupture. We recall that the normal stress differences are defined by

$$
N_{1} \equiv \Sigma_{z z}-\Sigma_{y y}
$$




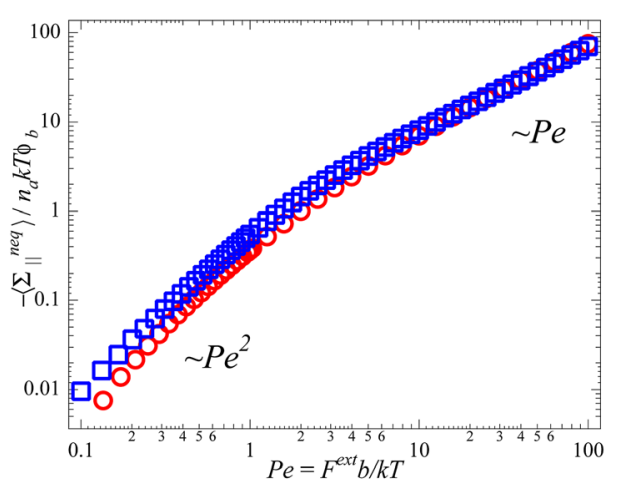

(a) Longitudinal normal stress

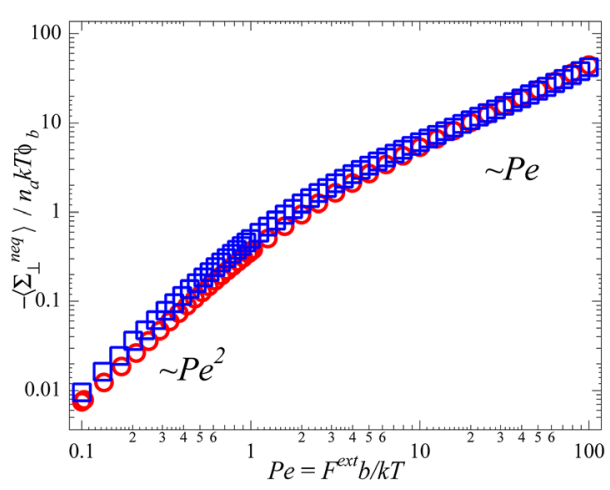

(b) Transverse normal stress

FIG. 2. Normal stress scaled by $n_{a} k T$ and volume fraction $\phi_{b}$ (size ratio $a / b=1$ ). Red circles represent the micromechanically derived stress (7): (○) $(3 / \pi) \boldsymbol{n} n g^{\text {neq }}(\boldsymbol{r}) d \Omega$. Blue squares represent the right-hand side of Eq. (28): ( $\square)\left(2-\eta^{\text {micro }} / \eta \phi_{b}\right)\left(\boldsymbol{D}^{\text {micro }} / D_{a} \phi_{b}\right)+\frac{1}{3} \operatorname{tr}\left(\boldsymbol{D}^{\text {micro }} / D_{a} \phi_{b}\right) \boldsymbol{I}$. Longitudinal components of both quantities shown in (a) and transverse components shown in (b).

$$
N_{2} \equiv \Sigma_{y y}-\Sigma_{x x}
$$

where $z$ is the direction of probe forcing, and $x$ and $y$ are the two orthogonal axes. According to Eq. (28), the first normal stress difference is

$$
-\frac{\left\langle N_{1}^{\text {neq }}\right\rangle}{n_{a} k T \phi_{b}}=\frac{D_{\|}^{\text {micro }}-D_{\perp}^{\text {micro }}}{D_{a} \phi_{b}}\left[2-\frac{\eta_{F}^{\text {micro }}}{\eta \phi_{b}}\right],
$$

and the second normal stress difference is zero,

$$
-\frac{\left\langle N_{2}^{\mathrm{neq}}\right\rangle}{n_{a} k T \phi_{b}}=0 .
$$

Figure 3(a) shows the right-hand side of Eq. (33) - the flux expression for the normal stress difference-compared to the left-hand side as computed via the micromechanically derived stress (7), where the former is denoted by open squares; the latter results were obtained both numerically (open circles) and analytically in the limit of small and large $P e$ (solid lines). Agreement is very good, although numerical resolution at small $P e$ can give rise to the small $<O\left(P e^{4}\right)$ difference shown. For comparison, the first-look theory [the right-hand side of Eq. (8)] is shown (cross symbols) in the same plot where it can be seen that the agreement is much improved. For strong forcing, $\left\langle N_{1}^{\text {neq }}\right\rangle$ scales linearly in the forcing $P e$. For weak forcing, $P e \ll 1$, Brownian motion easily restores isotropy to the microstructure around the probe, and $\left\langle N_{1}^{\text {neq }}\right\rangle$ vanishes as $P e^{4}$ as $P e \rightarrow 0$. Due to the axisymmetric structure around the probe, the second normal stress difference is zero for all $P e$. The solid lines shown in the figure are the analytical solution of Eq. (7) for asymptotically weak and strong forcing, showing excellent agreement between the proposed relation and the traditional micromechanical approach.

While normal stress differences indicate how a soft object might elongate, the osmotic pressure indicates how a compressible object might shrink or expand. The osmotic pressure is the trace of the probe-phase stress tensor; the corresponding nonequilibrium contribution is $\left\langle\Pi^{\text {neq }}\right\rangle / n_{a} k T=-\boldsymbol{I}:\left\langle\Sigma^{\text {neq }}\right\rangle / 3$, which is 


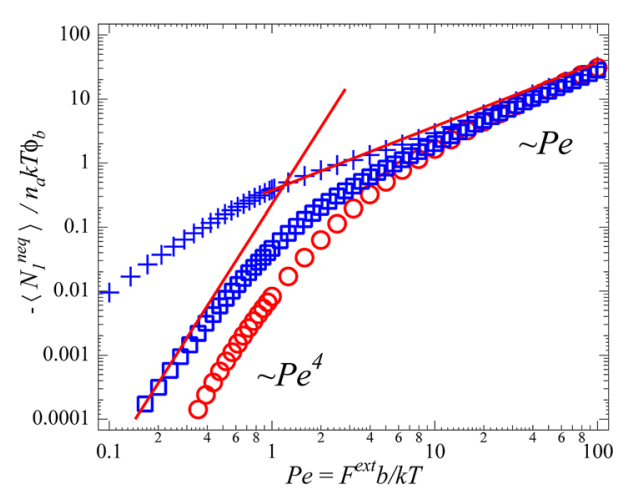

(a) First normal stress difference

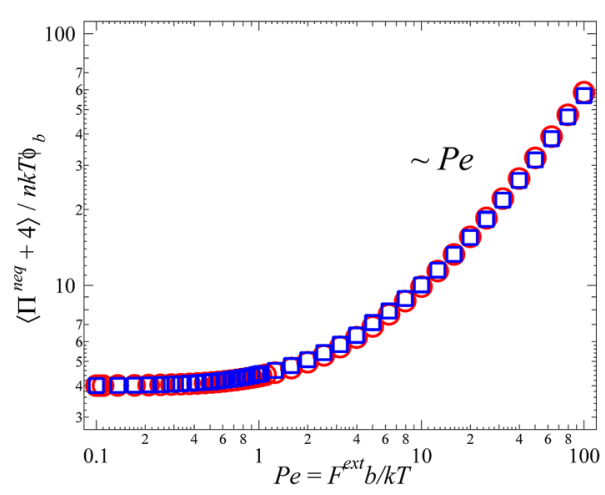

(b) Osmotic pressure

FIG. 3. First normal stress difference and osmotic pressure, scaled by $n_{a} k T$ and volume fraction $\phi_{b}(a / b=1)$. (a) Red symbols represent the micromechanically derived stress from Eq. (7): $(\bigcirc)$ numerical solution and $(-)$ analytical solutions (small and large $P e$ ) of $(3 / \pi) \int \boldsymbol{n} \boldsymbol{n} g^{\text {neq }}(\boldsymbol{r}) d \Omega$. Blue squares represent the right-hand side of Eq. (33): $(\square)-\left\langle N_{1}^{\text {neq }}\right\rangle / n_{a} k T \phi_{b}=\left[\left(D_{\|}^{\text {micro }}-D_{\perp}^{\text {micro }}\right) / D_{a} \phi_{b}\right]\left(2-\eta_{F}^{\text {micro }} / \eta \phi_{b}\right)$. Blue crosses represent the firstlook theory [Eq. (8)]: $(+)-\left\langle N_{1}^{\text {neq }}\right\rangle / n_{a} k T \phi_{b}=\left(D_{\|}^{\text {micro }}-D_{\perp}^{\text {micro }}\right) / D_{a} \phi_{b}$. (b) Red circles represent the micromechanically derived osmotic pressure from Eq. (7): (O) $\left\langle\Pi^{\text {neq }}\right\rangle / n_{a} k T \phi_{b}=(1 / \pi) \operatorname{tr} \int \boldsymbol{n} \boldsymbol{n} g^{\text {neq }}(r) d \Omega$. Blue squares from right-hand side of Eq. (35): ( $\square)\left\langle\Pi^{\text {neq }}\right\rangle / n_{a} k T \phi_{b}=\left[\left(D_{\|}^{\text {micro }}+2 D_{\perp}^{\text {micro }}\right) / 3 D_{a} \phi_{b}\right]\left(3-\eta_{F}^{\text {micro }} / \eta \phi_{b}\right)$.

$$
\frac{\left\langle\Pi^{\mathrm{neq}}\right\rangle}{n_{a} k T \phi_{b}}=\frac{1}{3} \frac{D_{\|}^{\text {micro }}+2 D_{\perp}^{\text {micro }}}{D_{a} \phi_{b}}\left[3-\frac{\eta_{F}^{\text {micro }}}{\eta \phi_{b}}\right] .
$$

The osmotic pressure is plotted as a function of the imposed force in Fig. 3(b), alongside the micromechanical result from Eq. (7). The results are indistinguishable. Expression (35) shows that the osmotic pressure may be determined by monitoring mean and meansquare motion of a single probe particle.

While expression (28) applies to the nonlinear microrheology of a dilute dispersion of colloids, the arguments offered to derive it are sufficiently general as to allow inclusion of other parameters that drive microstructural deformation and migration, e.g., electrostatic or hydrodynamic interactions. One need only determine the mean and mean-square motion of a probe in order to determine viscosity, diffusivity, and the stress tensor.

\section{B. Brownian dynamics simulation}

An alternative approach to studying particle behavior in colloidal dispersions is to examine the detailed dynamics of the individual particles. The dynamics of probe and bath-particle motion are governed by the Langevin equation, a force balance that includes Brownian, external, hydrodynamic, and other interparticle forces. In the present case, this balance reads

$$
\mathbf{0}=\boldsymbol{F}^{H}+\boldsymbol{F}^{\mathrm{ext}}+\boldsymbol{F}^{B}+\boldsymbol{F}^{P},
$$

where the left-hand side is zero because inertia is not important for colloidal dispersions. Periodic replication of the cell simulates an infinite domain. The hydrodynamic force is given by $\boldsymbol{F}^{H}=-6 \pi \eta a \boldsymbol{U}$ and the external force $\boldsymbol{F}^{\mathrm{ext}}=\mathbf{0}$ for all particles except the probe, for which it is prescribed. The interparticle force is modeled by a hard-sphere potential (cf. Appendix A), $\boldsymbol{F}^{P}=\boldsymbol{F}^{H S}$. The Brownian force in Eq. (36) is described by 


$$
\overline{\boldsymbol{F}^{B}}=0 \quad \overline{\boldsymbol{F}^{B}(0) \boldsymbol{F}^{B}(t)}=2 k T\left(6 \pi \eta a_{i}\right) \boldsymbol{I} \delta(t) .
$$

Here, the overbar denotes a statistical average indicating a random forcing and $\delta(t)$ is the Dirac delta function; $a_{i} \equiv a$ for the probe and $a_{i} \equiv b$ for a bath particle. This expression is integrated over a time step $\Delta t$ and solved for the total displacement. Brownian and hard-sphere displacements are scaled as $\Delta X \sim \sqrt{\left(2 k T / 6 \pi \eta a_{i}\right) \Delta t}$, and the dimensionless forcing is given by $P e=F^{\mathrm{ext}} /(k T / b)$. When $P e \gtrsim 1$, time is rescaled advectively in order to resolve the larger displacements occurring over a given time interval. To begin, a probe of size $a$ is placed among a randomly distributed bath of particles of size $b$ in the simulation cell. At each time step, every particle is given a randomly directed Brownian displacement, simulating a continuum Newtonian solvent of viscosity $\eta$. The probe is also displaced at each time step in the direction of the external force $\hat{\boldsymbol{F}}^{\text {ext }}$. When two particles contact one another, the hard-sphere collision is treated via a "potential-free" algorithm [Heyes and Melrose (1993); Carpen and Brady (2005)], where the overlap between two particles is prevented by separating the colliding pair along their line of centers until they are no longer in contact. The collision contributes to the particle's velocity and to the average stress in the suspension. For a complete description of Brownian dynamics of active microrheology, see Carpen and Brady (2005).

The nonequilibrium contribution to the particle stress due to a collision between the probe and a bath particle is $\left\langle\Sigma^{\text {neq }}\right\rangle=-n_{a}\left\langle\boldsymbol{r} \boldsymbol{F}^{P}\right\rangle$ where the angle brackets $\langle\cdot\rangle$ indicate an average over the duration of the simulation. The hard-sphere collision stresslet $-\boldsymbol{r} \boldsymbol{F}^{P}$ was measured at each time step, and averaged over the steady-state portion of the simulation. Here, $\boldsymbol{r}$ is the collisional displacement along the line of centers of the colliding particles. Simulations were conducted for a range of $P e$ and volume fraction $\phi_{b}$, with 280 simulations per parameter combination. The average stress for each simulation was then averaged over all 280 simulations.

The first normal stress difference $\left\langle N_{1}^{\text {neq }}\right\rangle=n_{a}\left\langle\left(r_{z} F_{z}-r_{x} F_{x}\right)\right\rangle=n_{a}\left\langle\left(r_{z} F_{z}-r_{y} F_{y}\right)\right\rangle$ is plotted in Fig. 4 as a function of $P e$ for a dilute bath. (A dilute bath is achieved by placing

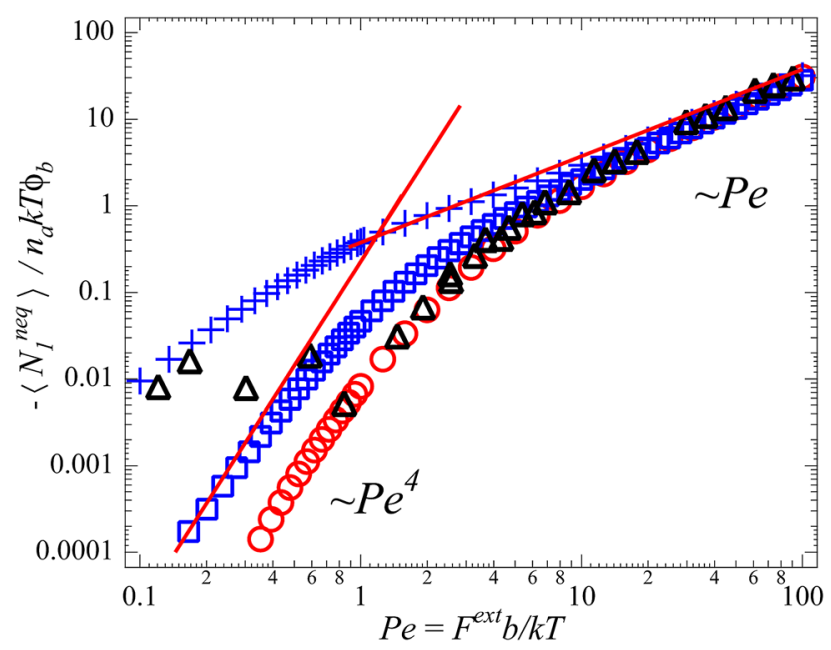

FIG. 4. First normal stress difference. $(\triangle)$ Brownian dynamics simulation results (each triangle represents 280 simulations; error bars are on the order of marker size.) Red symbols represent the micromechanically derived stress from Eq. (7): ( $\bigcirc$ ) numerical solution and $(-)$ analytical solutions (small and large $P e$ ) of $(3 / \pi) \int \boldsymbol{n} \boldsymbol{n} g^{\text {neq }}(\boldsymbol{r}) d \Omega$. Blue squares represent the right-hand side of Eq. (33): ( $\left.\square\right)-\left\langle N_{1}^{\text {neq }}\right\rangle / n_{a} k T \phi_{b}$ $=\left[\left(D_{\|}^{\text {micro }}-D_{\perp}^{\text {micro }}\right) / D_{a} \phi_{b}\right]\left(2-\eta_{F}^{\text {micro }} / \eta \phi_{b}\right)$. For comparison, the blue crosses represent the initial theory-the right-hand side of Eq. (8): $(+)-\left\langle N_{1}^{\text {neq }}\right\rangle / n_{a} k T \phi_{b}=\left(D_{\|}^{\text {micro }}-D_{\perp}^{\text {micro }}\right) / D_{a} \phi_{b}$. 
a probe particle amongst many "ideal-gas" bath particles in the cell-i.e., only probebath particle collisions occur, and the bath particles simply pass through each other. Since the bath particles do not directly see each other, they have no size except when they encounter the probe. It is their number concentration $n_{b}$, the contact length scale $(a+b)$ and the Brownian diffusivities $D_{a}$ and $D_{b}$, which govern the system dynamics. Varying the value of $\phi_{b}$ thus provides a means to compress the time required to obtain a sufficient number of collisions for statistical analysis [Zia and Brady (2010)]. The black triangles in Fig. 4 are thus for the dilute limit, $\phi_{b} \ll 1$.)

At small values of the Péclet number, the measured result for $\left\langle N_{1}^{\text {neq }}\right\rangle$ is close to zero in average value. For strong forcing, the first normal stress difference is proportional to the external force $P e$. The second normal stress difference $\left\langle N_{2}\right\rangle=n_{a}\left\langle\left(r_{y} F_{y}-r_{x} F_{x}\right)\right\rangle$, where we recall that $z$ is the direction of constant external force, is found to vanish on average. The average osmotic pressure $\langle\Pi\rangle=n_{b}\left\langle\left(r_{x} F_{x}+\right.\right.$ $\left.\left.r_{y} F_{y}+r_{z} F_{z}\right)\right\rangle / 3$ is plotted in Fig. 5, where again we find close agreement between the micromechanical approach, the theory, and the measurements obtained via Brownian dynamics.

Concentrated suspensions were also studied via dynamic simulation, up to a volume fraction of bath particles $\phi_{b}=0.45$ (in contrast to dilute simulations, here, the probe and bath particles all interact with each other). The scaling in volume fraction $\phi_{b}$ appears to hold for much more concentrated systems as is seen in the plot of the first normal stress difference (Fig. 6) and in the plot of the osmotic pressure (Fig. 7). The good agreement adds weight to the supposition that Eq. (28) is general and not restricted to dilute colloidal dispersions. This important outcome has both practical and fundamental implications. In practice, the theory predicts that the relation (28) holds for a large range of concentrations, which opens a wide class of natural and engineered complex media for investigation. Fundamental implications that the mobility is independent of the volume fraction of bath particles are discussed in Sec. IX.

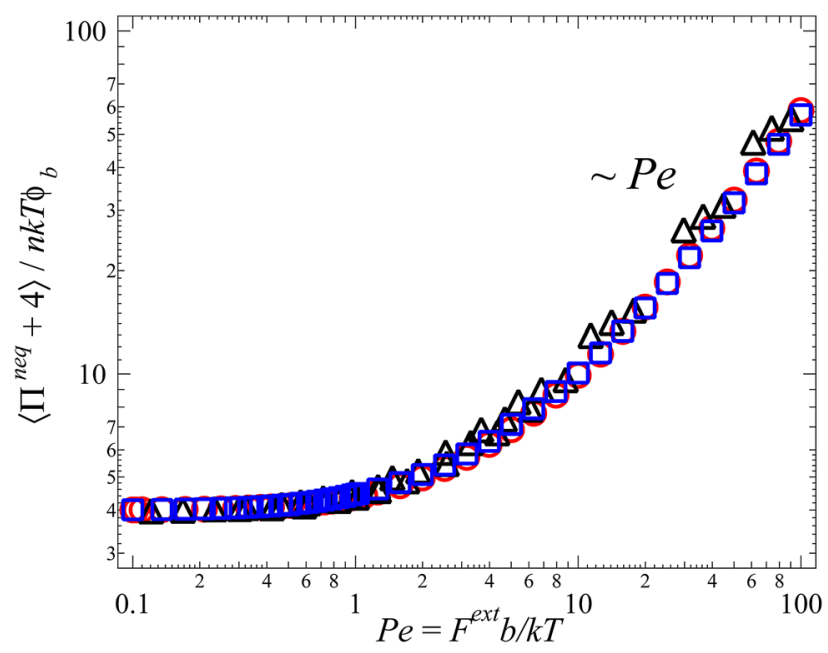

FIG. 5. Osmotic pressure. $(\triangle)$ Brownian dynamics simulation results (each triangle represents 280 simulations; error bars are on the order of marker size). Red circles represent the micromechanically derived stress from Eq. (7): $(\bigcirc)\left\langle\Pi^{\text {neq }}\right\rangle / n_{a} k T \phi_{b}=(1 / \pi) \operatorname{tr} \int \boldsymbol{n} \boldsymbol{n} g^{\text {neq }}(\boldsymbol{r}) d \Omega$. Blue open squares represent the right-hand side of Eq. (35): ( $\square)\left\langle\Pi^{\text {neq }}\right\rangle / n_{a} k T \phi_{b}=\left[\left(D_{\|}^{\text {micro }}+2 D_{\perp}^{\text {micro }}\right) / 3 D_{a} \phi_{b}\right]\left(3-\eta_{F}^{\text {micro }} / \eta \phi_{b}\right)$. 


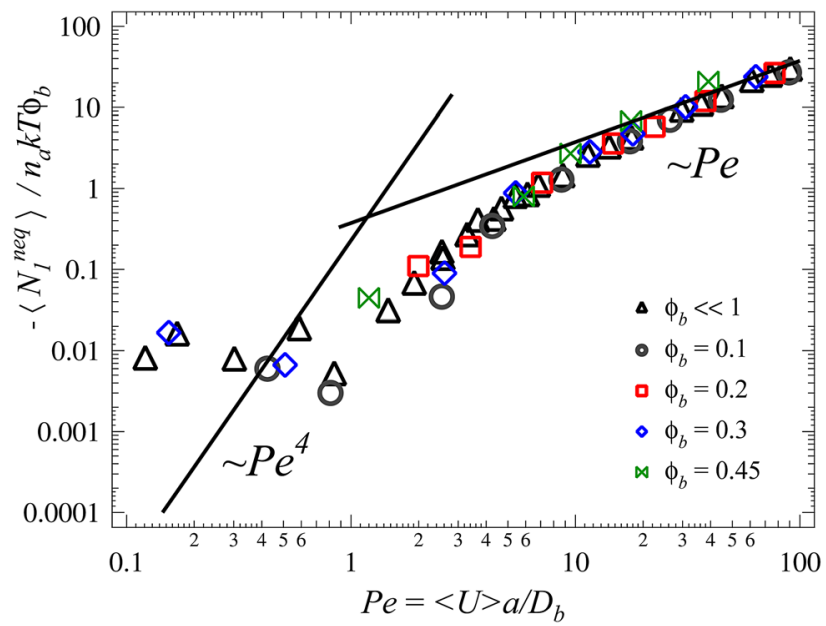

FIG. 6. Concentrated dispersions: First normal stress difference scaled by $n_{a} k T$ and volume fraction of bath particles $\phi_{b}$, all by Brownian dynamics simulation. Volume fractions as shown in legend in figure.

\section{MICRORHEOLOGY AND SHEAR MACRORHEOLOGY: A COMPARISON}

There have been a number of previous studies of normal stresses for colloidal dispersions undergoing bulk motion such as shearing or extensional flow. Theoretical investigations of dilute suspensions in simple shear were conducted analytically by Brady and Vicic (1995) for small $P e$; by Brady and Morris (1997) at large $P e$; and numerically for arbitrary $\mathrm{Pe}$ [Bergenholtz et al. (2002)], all with and without hydrodynamic interactions. Brownian dynamics simulations for dilute and concentrated sheared suspensions were conducted by Foss and Brady (2000a); they also studied the effect of hydrodynamic interactions via Stokesian dynamics simulation [Foss and Brady (2000b)] for a dispersion undergoing simple shear. Sami (1996) conducted similar studies in the case of an extensional flow. A brief discussion of the results is given below. For each of the studies

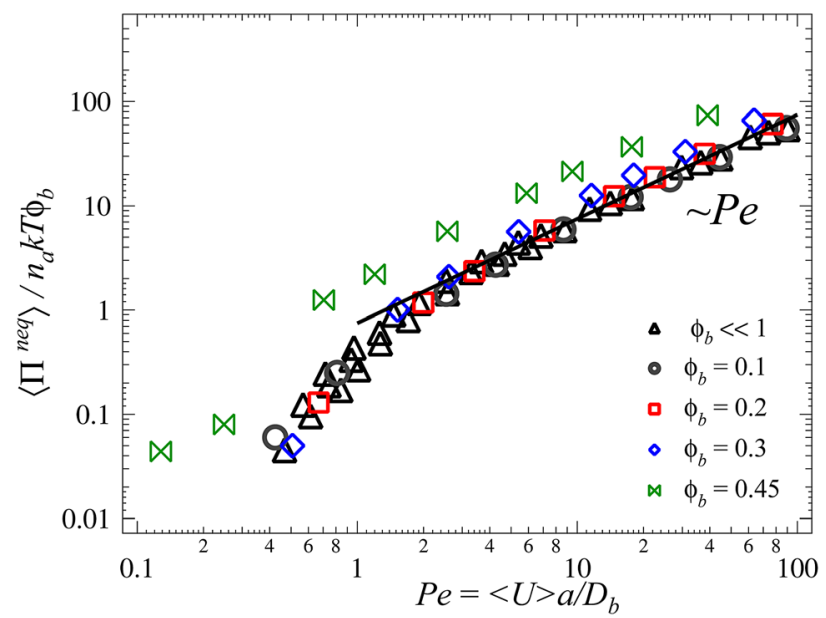

FIG. 7. Concentrated dispersions: Nonequilibrium osmotic pressure scaled by $n_{a} k T$ and volume fraction of bath particles $\phi_{b}$, all by Brownian dynamics simulation. Volume fractions as shown in legend in figure. 
considered, colloidal particles interact through hydrodynamic and interparticle forces which may be both short- and long-ranged, as discussed in Appendix A.

In a study of low-Pe behavior, Brady and Vicic (1995) found that the first contribution to non-Newtonian behavior in a suspension undergoing shear in the absence of hydrodynamic interactions is at $O\left(P e^{2} \phi^{2}\right)$, in both the normal stress differences and the viscosity (shear thinning), with $N_{1}>0$ and $N_{2}<0$. The numerical study of Bergenholtz et al. (2002) confirmed the low-Pe results of Brady and Vicic: Normal stress differences do indeed arise in the absence of hydrodynamic interactions, because hard-sphere repulsion allows particles to cross streamlines, giving rise to random motion and breaking the reversibility required in Stokes' flow. Comparison of these low-Pe, no-hydrodynamics results to those of the microrheology study shows both similarity and differences: In both macro and micro, the first order at which non-Newtonian rheology emerges is $O\left(P e^{2}\right)$ as expected for small departures from equilibrium. In macrorheology, both $N_{1}$ and $N_{2}$ are nonzero at small $P e$, whereas in microrheology the axisymmetry about the probe produces a zero second normal stress difference. This difference between the two flows is discussed in more detail below. It should be noted that the scaling in volume fraction $\phi_{b}$ is different in the two cases. While both macro and micro measure a pair-level contribution to the stress, in macrorheology, pair-level interactions arise at $O\left(\phi_{b}^{2}\right)$ as two particles must interact in the flow. But in microrheology, interactions are studied in a frame of reference relative to the probe and so its interaction with another particle is $O\left(\phi_{b}\right)$. This natural scaling difference is thus simply understood.

Brady and Morris (1997) studied the opposite extreme of very strong shear forcing for dilute suspensions; they determined the diffusion tensor and the stress tensor, finding $N_{1} / n k T=0$ and $N_{2} / n k T \phi^{2}=-(16 \pi P e / 15)$. They also noted that the connection between diffusion and stress in their results, namely, that $\partial \Sigma / \partial \phi \propto \boldsymbol{D}$. Bergenholtz et al. (2002) extended this study over the full range of $P e$, finding that at high $P e, N_{1} / n k T \phi^{2} \sim$ $P e>0$ and $N_{2} / n k T \phi^{2} \sim P e<0$, with $N_{2} / n k T \phi^{2} \rightarrow-12 \pi P e / 5$ as $P e \rightarrow \infty$.

Biaxial extensional flow was studied by Sami (1996) via Stokesian dynamics simulations for concentrated colloidal dispersions. Here, analogously to the present study, normal stresses and their differences are related to the viscosity and to microstructural asymmetry. Because the normal stress differences are linear in the extensional viscosity, his results also predict the first normal stress difference, where he finds that $N_{1} / n k T \phi^{2} \sim$ $P e$ and $N_{2} / n k T \phi^{2} \sim P e$ for large $P e$.

Figure 8 compares the normal stresses in microrheology and shear macrorheology for dilute colloids on the plane normal to the flow direction. Two sets of longitudinal stress data are shown: The open squares for microrheology correspond to Eq. (28), and the solid black line gives the longitudinal stress in shear macrorheology. Both show the same scaling in $P e$ for the entire range studied, but with a quantitative difference, or offset. The fundamental difference between the microstress and macrostress is that in macrorheology, all viscous stresses are in the off-diagonal components, whereas in microrheology, viscous stresses are normal.

Figure 9 gives a comparison between the macro and micro normal stresses on the plane transverse to the flow, where we first note that one major difference between the two flows is their symmetry properties. In the case of microrheology, the structure around the probe is axisymmetric [Fig. 12(a)] and so there is no distinct direction orthogonal to the flow. On the other hand, in macrorheology the structure around a tracer is skew symmetric [Fig. 12(b)], so the stress on a plane along the velocity-gradient direction is different than that along the vorticity direction [Bergenholtz et al. (2002)]. As shown in Fig. 9, despite this fundamental difference, the similarity between micro and macro is very strong: The same scaling in $P e$ is evident for the full range of $P e$ and the quantitative 


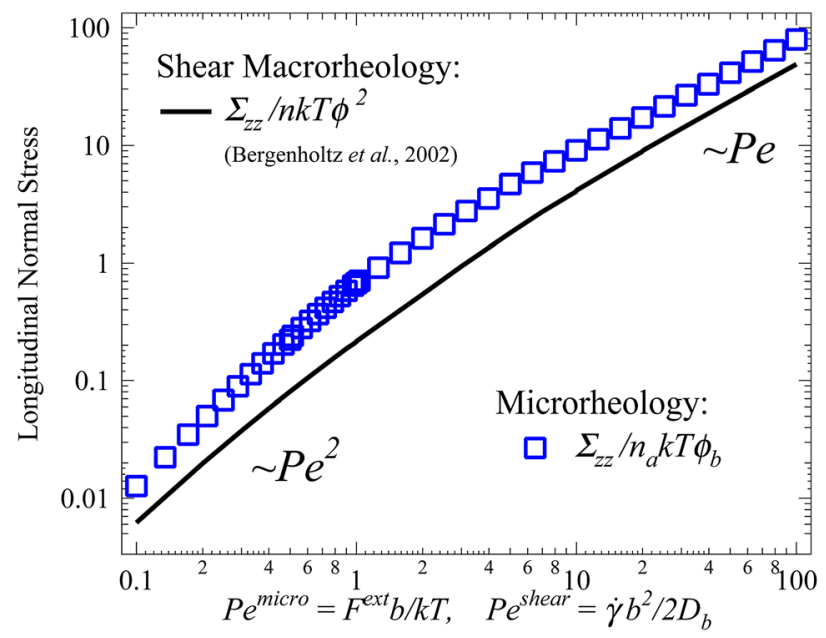

FIG. 8. Macrorheology versus microrheology: normal stress on the plane normal to the flow direction. Macrorheology: solid line (-) by Bergenholtz et al. (2002) for simple shear of dilute colloidal dispersion of hard spheres. Microrheology: blue open squares correspond to the right-hand side of Eq. (28): ( $\square$ ) $\left\langle\Sigma_{z z}\right\rangle / n_{a} k T \phi_{b}=\left(2-\eta_{F}^{\text {micro }} / \eta \phi_{b}\right)\left(D_{z z}^{\text {micro }} / D_{a} \phi_{b}\right)+\operatorname{tr}\left(\boldsymbol{D}^{\text {micro }} / D_{a} \phi_{b}\right) / 3$.

agreement is good. But the fundamental difference between the two microstructures is seen here: the second normal stress difference is zero in microrheology, and $\mathrm{O}(\mathrm{Pe})$ for strong forcing in macrorheology.

The first normal stress difference is similar for the two flows, as shown in Fig. 10. The solid line represents data for shear macrorheology [Bergenholtz et al. (2002)]; the open squares reflect the full equation (33) for microrheology, while the crosses are the same result but with the viscous stress [the second term in Eq. (28)] removed, as suggested above. Agreement between all three sets of data is fair at high $P e$, where viscous stress does not matter for the microrheology tensor. But at moderate and low $P e$, agreement

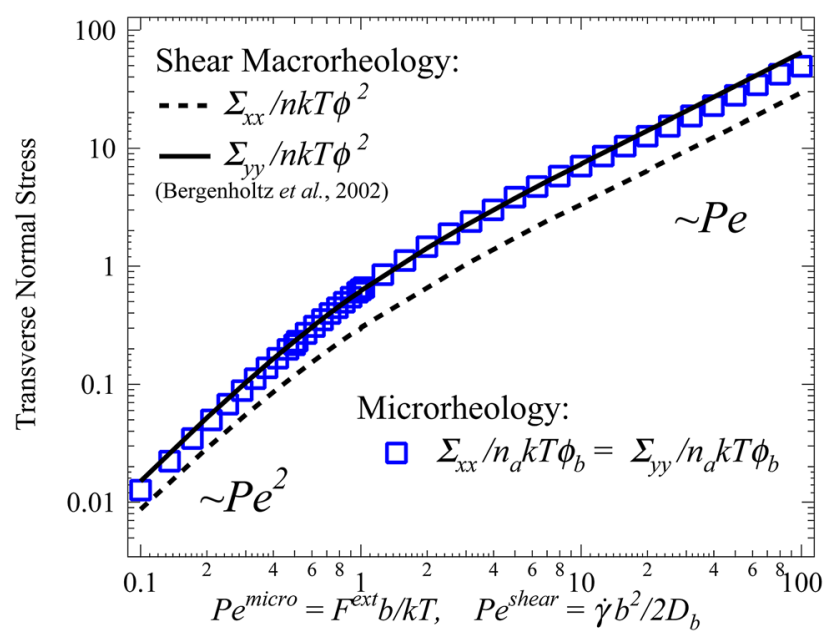

FIG. 9. Macrorheology versus microrheology: normal stress on the plane transverse to flow direction. Macrorheology: solid, dashed lines by Bergenholtz et al. (2002) for simple shear of dilute colloidal dispersion [solid line: (-) velocity-gradient direction; dashed line: (- - ) vorticity direction]. Microrheology: blue open squares correspond to the right-hand side of Eq. (28): ( $\square)\left\langle\Sigma_{x x}\right\rangle / n_{a} k T \phi_{b}=\left(2-\eta_{F}^{\text {micro }} / \eta \phi_{b}\right)\left(D_{x x}^{\text {micro }} / D_{a} \phi_{b}\right)$ $+\operatorname{tr}\left(\boldsymbol{D}^{\text {micro }} / D_{a} \phi_{b}\right) / 3$. 


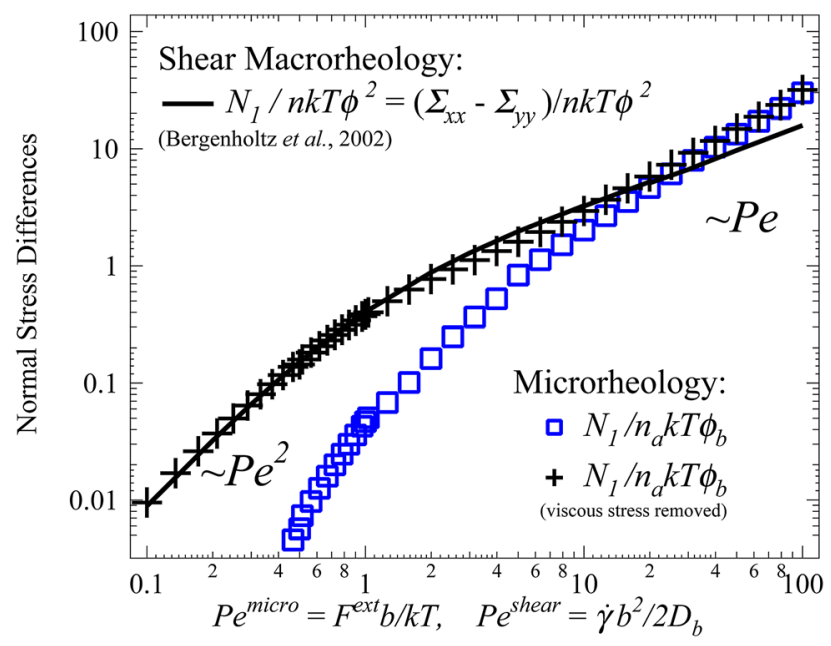

FIG. 10. Macrorheology versus microrheology: normal stress differences. Solid line (-) represents numerical results by Bergenholtz et al. (2002) for simple shear of dilute colloidal dispersion. Blue open squares are for nonlinear microrheology [Eq. (33)]: ( $\square)\left[\left\langle N_{1}\right\rangle / n_{a} k T \phi_{b}=\left(D_{\|}^{\text {micro }}-D_{\perp}^{\text {micro }}\right) / D_{a} \phi_{b}\right]\left(2-\eta_{F}^{\text {micro }} / \eta \phi_{b}\right)$. Black crosses: Eq. (33) without viscous stress: $(+)\left(D_{\|}^{\text {micro }}-D_{\perp}^{\text {micro }}\right) / D_{a} \phi_{b}$.

between macro and micro is poor, when the viscous stress is included. A comparison between macrorheology and microrheology $\left\langle N_{1}\right\rangle$ without the viscous normal stress is also plotted (cross symbols) and shows much-improved agreement.

The final comparison between the two flows is the osmotic pressure which, for a dilute bath, is plotted in Fig. 11. For the microrheology results, the full stress tensor [Eq. (35)] is used. Here, we see strong qualitative and quantitative agreement over the full range of $P e$. It is interesting that the agreement in behavior for the osmotic pressure is so strong when the stress tensor components individually showed at least quantitative

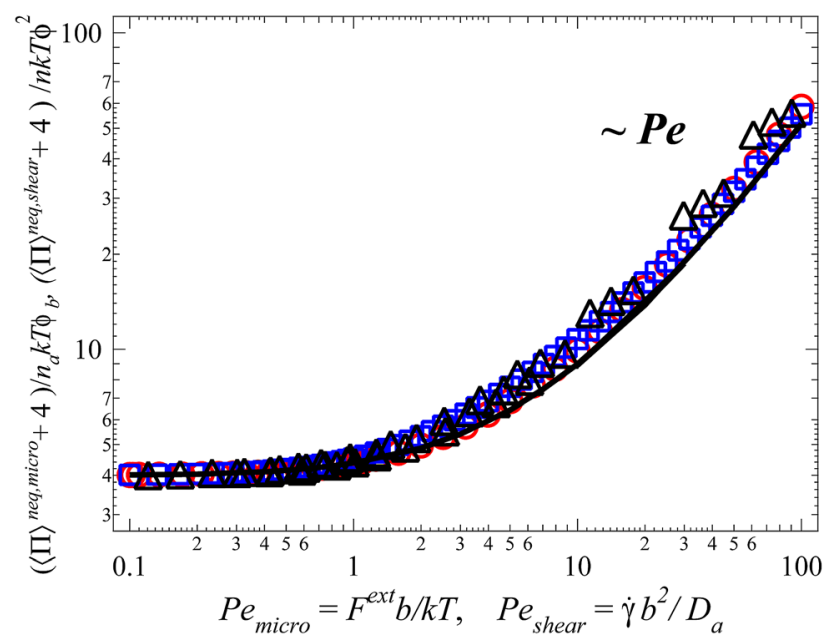

FIG. 11. Macrorheology versus microrheology (dilute system): osmotic pressure. Macrorheology: (-) numerical results by Bergenholtz et al. (2002) for simple shear of dilute colloidal dispersion of hard spheres. Microrheology: $(\triangle)$ Brownian dynamics simulation results (each triangle represents 280 simulations; error bars are on the order of marker size); red circles represent the micromechanically derived stress from Eq. (7): $(\bigcirc)$ $\left\langle\Pi^{\text {neq }}\right\rangle / n_{a} k T \phi_{b}=(1 / \pi) \operatorname{tr} \int \boldsymbol{n} \boldsymbol{n} g^{\text {neq }}(\boldsymbol{r}) d \Omega$; blue open squares represent the right-hand side of Eq. (35): $(\square)$ $\left\langle\Pi^{\text {neq }}\right\rangle / n_{a} k T \phi_{b}=\left[\left(D_{\|}^{\text {micro }}+2 D_{\perp}^{\text {micro }}\right) / 3 D_{a} \phi_{b}\right]\left(3-\eta_{F}^{\text {micro }} / \eta \phi_{b}\right)$. 


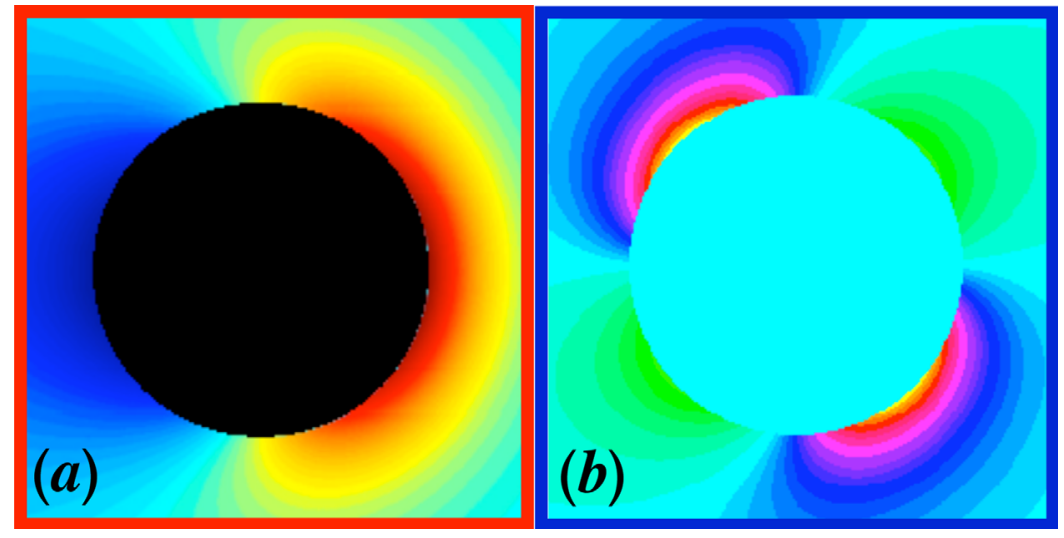

FIG. 12. Comparison of nonequilibrium microstructure around a probe particle in (a) microrheology, in a plane in the line of the external force passing through the origin of the probe [Zia and Brady (2010)]; and (b) simple shear flow [Bergenholtz et al. (2002)] viewing the velocity/velocity-gradient plane. Red and yellow areas indicate regions of strong and moderate probability accumulation, respectively, while dark blue and blue indicate regions of strong and moderate depletion, respectively. Colloidal dispersion is dilute and $P e=5$ for both plots.

disagreement. In order to understand why this agreement exists, it is useful to consider the mathematical underpinning of the osmotic pressure: It represents the invariants of the stress tensor. In one flow, the deformed microstructure is axisymmetric (micro) while in the other flow it is skew symmetric (macro), as shown in Fig. 12. A rotation of quadrants can make their structures nearly identical. And because the stress is the second moment of the microstructure, the sign does not change in such a transformation. Further, a diagonalization of the macrostress tensor using its eigenvalues would move the viscous stress components to the diagonal; because the eigenvalues are preserved in such a transformation, the osmotic pressure reflected in the transformed macrostress tensor should bear similarity to that of the microstress tensor, if one considers the similarity in structure after a quadrant rotation as noted above. This view may also contribute understanding as to why the overall agreement between microrheology and macrorheology is so strong.

\section{PROBE/BATH-PARTICLE INTERACTIONS VERSUS BATH/BATH-PARTICLE INTERACTIONS}

Up to now we have examined the nonequilibrium particle stress by driving the bath from equilibrium with a Brownian probe particle and measuring the collisions suffered by the probe due to interactions with the bath particles. In a dilute bath, interactions between the bath particles themselves make an $O\left(\phi_{b}^{2}\right)$ contribution to the nonequilibrium osmotic pressure, which is small for a dilute bath; hence, the probe-phase pressure dominates the pressure of all the particles (probe plus background bath particles).

This simple scaling prediction is borne out by Brownian dynamics simulations, in which the bath particles interact via excluded-volume interactions. The contribution to the osmotic pressure of both probe and bath particles was monitored during simulations with volume fraction of bath particles $\phi_{b}=0.1$. The stress measured by probe/bath-particle collisions, $\langle\Sigma\rangle / n_{a} k T \phi_{b}$, and that for bath/bath-particle collisions, which shall be denoted $\langle\Sigma\rangle_{b b} / n_{b} k T \phi_{b}$, was recorded. The osmotic pressure thus obtained, as a function of $P e$, is shown alongside those for the dilute bath in Fig. 13. 


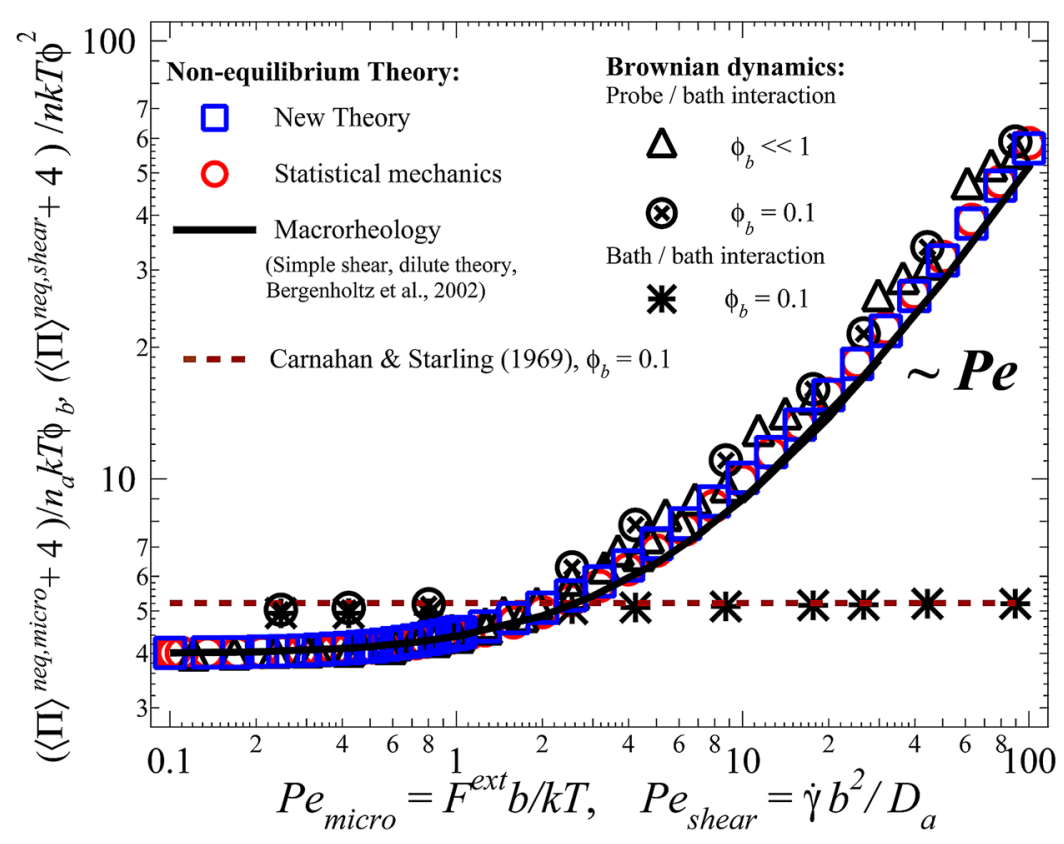

FIG. 13. Osmotic pressure. Comparison of contribution due to bath-bath collisions $(*)$ versus probe-bath collisions $(\otimes)$ in microrheology via BD simulation at $\phi_{b}=0.1$. Also shown: comparison of dilute microrheology $(\triangle)$ to macrorheology $(-)$ in a dilute bath of hard spheres undergoing simple shear. Brown broken line: equilibrium osmotic pressure via Carnahan-Starling equation of state at $\phi_{b}=0.1$ : (- - ). Red circles represent the micromechanically derived osmotic pressure from Eq. (7): ( $\bigcirc)\left\langle\Pi^{\text {neq }}\right\rangle / n_{a} k T \phi_{b}=(1 / \pi) \operatorname{tr} \int \boldsymbol{n} \boldsymbol{n} g^{\text {neq }}(\boldsymbol{r}) d \Omega$; blue open squares represent the right-hand side of Eq. (35): ( $\square)\left\langle\Pi^{\text {neq }}\right\rangle / n_{a} k T \phi_{b}=\left[\left(D_{\|}^{\text {micro }}+2 D_{\perp}^{\text {micro }}\right) /\right.$ $\left.3 D_{a} \phi_{b}\right]\left(3-\eta_{F}^{\text {micro }} / \eta \phi_{b}\right)$.

While the osmotic pressure measured by the probe, $\langle\Pi\rangle / n_{a} k T$, increases linearly with $P e$, the osmotic pressure due to the bath/bath-particle interactions $\langle\Pi\rangle_{b b} / n_{b} k T$ remains at the equilibrium value regardless of $P e$ and is indistinguishable from the equilibrium osmotic pressure predicted by the Carnahan-Starling equation of state [Carnahan and Starling (1969)]. The behavior seen in the figure reflects the fact that most of the bath particles interact with each other due to Brownian motion only, whereas the probe and bath particles interact due to the external forcing. In order to understand if this matches what one would expect from traditional macrorheology experiments, the dilute theory results for a bath undergoing simple shear [Bergenholtz et al. (2002)] are plotted alongside the microrheology results in Fig. 13; the two agree.

\section{A “NONEQUILIBRIUM EQUATION OF STATE”}

Our work suggests that nonlinear microrheology may provide more than a microscale technique for interrogating complex fluids. It provides a window through which one can view the atomic (microstructural) world. In 1906, Einstein invented "passive microrheology" to prove the atomic nature of matter. He proposed that if matter were indeed composed of atoms, then the motion of a small particle suspended in a fluid would move with the same random trajectories as the solvent particles. Combining the theory from kinetics, diffusion, and thermodynamics, he gave the relationship between fluctuation and dissipation at equilibrium, which showed that the diffusive motion of a small particle is indeed evidence of the existence of the atom. Perrin confirmed the theory with measurement in 1909. This is a profound conclusion, drawn by simply watching the motion of a 
single particle in a fluid. Together, theory and experiment produced the numerical value for Avogadro's number by relating the bulk temperature at the macroscale to the energy of individual particles at the microscale. Thus, the ideal-gas equation of state $P V=\bar{n} \mathcal{R} T$ (where $\bar{n}$ is the number of moles) became $P=n k T$ (where $n$ is the number density of particles). Inspired by this, we too watch a particle move in a complex fluid-but now for a system that is not at equilibrium. Here, by studying fluctuations away from equilibrium, we propose an analogous nonequilibrium relation between fluctuation and dissipation, Eq. (29).

Kinematically the diffusivity is given by the sum of correlations in velocity fluctuations over time,

$$
D \sim \int U^{\prime} U^{\prime} d t \sim\left(U^{\prime}\right)^{2} \tau
$$

where $\tau$ is the characteristic time scale for the decay in the velocity correlations. For Brownian motion, $\left(U^{\prime}\right)^{2} \sim k T / m$ and $\tau \sim m / 6 \pi \eta a$, where $m$ is the particle mass, and the usual Stokes-Einstein diffusivity is recovered. But more generally, the magnitude of the velocity fluctuations defines the temperature, $T \sim U^{\prime} U^{\prime}$, whence

$$
\Sigma \sim n_{a} C T^{\mathrm{eff}} .
$$

Here, we have defined the proportionality factor $C \equiv\left(M^{\text {neq }}\right)^{-1} \tau$ and the "effective temperature" of the dispersion is given by particle velocity fluctuations, $T^{\text {eff }} \equiv\left\langle U^{\prime} U^{\prime}\right\rangle$. The proportionality of the stress to $\left\langle U^{\prime} U^{\prime}\right\rangle$ prompts us to interpret the stress, or osmotic pressure, as the energy density of the particle phase. Equation (39) can thus also be interpreted as a nonequilibrium equation of state for the particle phase, with $C$ the factor of proportionality that connects the microscopic energy density to the macroscale effective temperature. One may also view the time dependence $\tau$ of the factor $C$ as the time scale over which the energy stored by the microstructure is dissipated [Zia and Brady (2012)]. The proposed generality of this relation may be important in unifying the analysis, understanding, and treatment of a wide class of soft materials.

\section{EXPERIMENTAL MEASUREMENT}

We have presented a theoretical framework that relates the particle-phase stress of a colloidal dispersion to the mean and mean-square displacement of a probe driven through the suspension, which we hope will be of practical use in making physical measurements. As noted below, detailed knowledge of the embedding material is not required. In order to obtain the stress tensor, the only quantity which must be measured is the total displacement of the probe over time. From this, the average speed $\langle\boldsymbol{U}\rangle=d\langle\boldsymbol{x}\rangle / d t$ can be computed to obtain the microviscosity via the relation,

$$
\frac{\eta_{F}^{\text {micro }}}{\eta}=\frac{6 \pi \eta a}{\left\langle F^{\mathrm{ext}}\right\rangle_{F}}\langle U\rangle,
$$

where $\left\langle F^{\text {ext }}\right\rangle_{F}$ is a constant external force applied to the probe, and $\eta_{F}^{\text {micro }}$ is the microviscosity. From the total and mean displacement, one may then compute the microdiffusivity as

$$
\boldsymbol{D}^{\text {micro }}=\frac{1}{2} \frac{d}{d t}\left\langle\boldsymbol{x}^{\prime}(t) \boldsymbol{x}^{\prime}(t)\right\rangle
$$


where $\boldsymbol{x}^{\prime}(t) \equiv \boldsymbol{x}(t)-\langle\boldsymbol{x}\rangle(t)$ is the displacement from the mean as function of time and the angle brackets signify an average over realizations. We believe that the development of the relation (28) is sufficiently general as to apply to a very wide class of complex media, ranging from colloidal dispersions to gels to solids with suspended dislocations. Combining Eq. (28) with Eqs. (40) and (41), we propose that the stress in a general material can be obtained by measuring the mean and mean-square motion of a probe driven through the medium by a constant external force,

$$
-\frac{\Sigma^{\text {neq }}}{n_{a} k T}=\left(2-\frac{\eta_{F}^{\text {micro }}}{\eta \phi_{b}}\right) \frac{\boldsymbol{D}^{\text {micro }}}{D_{a}}+\frac{1}{3} \operatorname{tr}\left(\frac{\boldsymbol{D}^{\text {micro }}}{D_{a}}\right) \boldsymbol{I} .
$$

It should be noted that for $P e \lesssim 1$, the strong influence of Brownian motion can make it difficult to detect the small differences between the longitudinal and transverse microdiffusivity, and hence between the corresponding normal stresses. It is thus recommended that the regime most suitable for experimental measurement using this analysis is $P e \gtrsim 1$.

\section{CONCLUSIONS}

We have presented a new approach for understanding and determining the stress in a dispersion of colloidal particles. Inspired by the equilibrium connection between osmotic pressure gradients and diffusion, it was proposed that diffusion is driven by stress gradients in nonequilibrium suspensions as well. Previous work has connected gradients in number density and chemical potential to collective diffusion [Einstein (1906); Batchelor (1972)]; these treatments applied to equilibrium Brownian suspensions. This connection holds appeal in that the simple measurement of mean-square displacement would then provide a measurement of suspension stress. It was thus proposed that the relation $\partial \boldsymbol{\Sigma} / \partial n \propto \boldsymbol{D}$ holds for nonequilibrium suspensions. The corresponding equation $\Sigma=-n \boldsymbol{R} \cdot \boldsymbol{D}$ was evaluated for the case of nonlinear microrheology; on the right-hand side of this relation is the force-induced diffusion, $\boldsymbol{D}^{\text {micro }}$, with the resistance tensor for the dilute dispersion $\boldsymbol{R}=6 \pi \eta a \boldsymbol{I}$. The left-hand side was computed as the second moment of the microstructure $g(\boldsymbol{r})$, where $g(\boldsymbol{r})$ is governed by a pair-Smoluchowski equation. In the comparison between this first-look approach and the micromechanical approach, the scaling dependence on $\mathrm{Pe}$ was correct, but quantitative agreement was not perfect. Taking a phenomenological view of the stress tensor, it was noted that the effect of viscous forces was not accounted for in $\Sigma$. Underlying assumptions for the model were examined to determine where physical processes may have been neglected. The primary feature of the equilibrium model limiting its generalization to nonequilibrium systems is that it was derived from an equation of state, while for nonequilibrium systems an equation of motion is required.

Cauchy's momentum balance was presented as the equation of motion of the suspension, where the inertial and time-dependent terms were discarded due to a vanishingly small Reynolds number. To consider the probe-particle stress only, an interactive force with the bath particles was necessary to account for the effect of the remaining medium on the probe particles. Several cases were examined in which the constitutive form of the interactive force corresponded to the relevant transport process. For equilibrium suspensions subjected to a particle-density gradient but no external body forces, Fickian diffusion was taken as the constitutive model for the flux arising due to the interactive force. Next, for no gradients but with an external body force-constant-velocity nonlinear 
microrheology - the interactive force is the mean advective drag of the microstructure. This important case indicates that advective, as well as diffusive, flux should be considered in the nonequilibrium system.

Before moving on to consider a more general system - the presence of both an external body force and particle-density gradients-it was noted that the thermal agitation of particles that gives rise to the isotropic osmotic pressure at equilibrium will also be present away from equilibrium, where the probes scatter actively off of the microstructure. Intuitively this should increase agitation and lead to a higher isotropic particle-phase pressure; that is, it should have the effect of an increased effective temperature in the suspension. Particle fluctuations due to scattering increase as flow strength increases, which can be linked to the velocity fluctuations $\boldsymbol{U}^{\prime}$ and so it was proposed that this $P e$-dependent isotropic pressure be constitutively modeled by the trace of the induced diffusion tensor.

Next, the case of constant-force nonlinear microrheology was considered, where the probe velocity can fluctuate. The freedom to fluctuate gives the probe the ability to adjust its speed when encountering regions of higher or lower density. The hindrance of the microstructure is smaller than that of the constant-velocity case, which has the effect of weakening the viscous resistance to its motion. That is, stress gradients drive advective flux of the probe, completing the nonequilibrium, coarse-grained model.

The total flux predicted by the theory was compared to known results obtained via a micromechanical approach and with a measurement of the stress via Brownian dynamics simulations. The normal stress differences were compared side-by-side among the three approaches, showing excellent agreement. Due to the axisymmetry of the microstructure about the axis of forcing, the second normal stress difference $\left\langle N_{2}\right\rangle=0$ for all $P e$. For large $P e$, the first normal stress difference scales linearly in the forcing and in the volume fraction of bath particles, $\left\langle N_{1}\right\rangle / n_{a} k T \sim P e \phi_{b}$. For very weak forcing, $P e \ll 1,\left\langle N_{1}\right\rangle$ vanishes as $\sim O\left(P e^{4}\right)$, because the Brownian motion of the bath particles easily restores deformation caused by the probe's motion, and the structure is nearly isotropic. One could expect instead that $\left\langle N_{1}\right\rangle$ should vanish as $P e^{2}$, as might be predicted from nearequilibrium, linear-response theory. But this is where the importance of normal viscous stresses plays a special role in microrheology. Without these, the decay in $\left\langle N_{1}\right\rangle$ for very weak forcing would indeed scale quadratically in $P e$. Although viscosity plays a role in the stress due to fluctuations, the viscous drag due to mean motion always acts to slow the probe; at small $P e$, the effect is a stronger suppression of the advective motion, which results in a stronger suppression of microstructural asymmetry.

The osmotic pressure was computed via the new theory and was also compared to the statistical mechanics and Brownian dynamics results, with excellent agreement. For large $P e$, the osmotic pressure scales linearly in the forcing and in the volume fraction of bath particles. For very weak forcing, $P e \ll 1$, the total nonequilibrium osmotic pressure $\Pi$ asymptotes to the value predicted by the Carnahan-Starling equation of state [Carnahan and Starling (1969)].

Although the proposed theory was constructed for and compared with dilute dispersions, the physical ideas underlying the theory should hold more generally; and indeed, it was shown that the proposed relation (28) also applies to much more concentrated colloidal dispersions. Higher concentrations of bath particles were studied via dynamic simulation and the same stress-migration relation was shown to be valid. This important result holds both practical and fundamental appeal. From a practical standpoint, the applicability of the theory to concentrated systems is critical to its use in many systems of interest, e.g., watery biophysical systems such as the crowded interior of the cell. From a fundamental standpoint, the linear dependence of the stress in volume fraction $\phi_{b}$ is intriguing; it implies that the mobility of the probe is independent of $\phi_{b}$. 
To give a physical picture of the importance of the normal stresses, one can imagine a soft particle, such as an organelle inside the cell, or such as a bubble, placed in a suspension. If the particle is compressible, then forcing it through the medium will cause it to shrink or expand - and possibly burst - due to the particle pressure. If the particle is soft, the normal stress differences that arise due to forcing will elongate it-possibly causing rupture. These effects have important implications in the consideration of nanoparticle drug delivery and the escape of viruses from the endosomal pathway, for example.

Microrheology need not-in fact should not-be viewed as a microscale version of traditional shear rheology. However, one can always ask whether the measurements obtained via microrheology correspond in a meaningful way with the bulk measurements obtained by shear macrorheology. For example, Khair and Brady (2006) demonstrated that the microviscosity recovers all of the important phenomenological behavior of a dilute colloidal dispersion under flow, for same-size particles- the same scaling in $P e$, the same onset of shear thinning and thickening, and in the limit of negligible hydrodynamics, the same terminal Newtonian plateau. In Sec. V, we compared the osmotic pressure results for microrheology with the dilute theory of Bergenholtz et al. (2002) for macrorheology, and found that the osmotic pressure measured by single-probe forcing matches quite well with the dilute shear rheology theory. This is actually somewhat surprising if one considers the dependence of stress on the contact value of the microstructure. The deformed microstructures of the two flows-simple shear and microrheology-are markedly different. One important difference became evident in the comparison of the individual elements of the stress tensors. The parallel and perpendicular diagonal elements of the stress tensor in macrorheology contain no viscous stress: viscosity is strictly deviatoric in simply sheared suspensions. In contradistinction, viscous stress in microrheology is normal and must appear on the diagonal of the stress tensor. When the viscous stress was removed from the theoretical prediction [the second term in brackets in Eq. (33)], very good agreement between the first normal stress difference in microrheology versus macrorheology was found.

It is interesting that the agreement in behavior for the osmotic pressure is so strong when the stress tensor components themselves showed some quantitative disagreement. Physically, the shear microstructure is skew symmetric about a test particle, while for microrheology it is axisymmetric. But if one refers to the microstructural sketches of simple shear versus microrheology in Fig. 12, one can imagine rotating the upper left quadrant of the shear microstructure to the upper right quadrant; the structures are then strikingly similar. Since the sign change would not matter in the second moment of this structure, it is not surprising then that at least the osmotic pressure is the same. This view may also contribute understanding as to why the overall agreement between microrheology and macrorheology is so strong for the case considered here.

An additional important consideration in the comparison of microrheology to macrorheology is the fact that microrheological flows are not viscometric: they do not produce statistically homogeneous flows and microstructures. The microstructure around a driven probe is statistically different than the structure away from the probe. The question then arises whether the suspension properties sampled by the probe reflect the bulk properties of the medium, as is the case in macrorheology. A similar concern arises in falling-ball rheometry. It has recently been proposed in the literature that the probe-phase stress is not the only contributor to overall particle-phase stress-that interactions between the bath particles themselves make a significant contribution [Squires (2008)]. A simple scaling analysis shows that bath/bath-particle interactions contribute only to $O\left(\phi_{b}^{2}\right)$, a negligible contribution for a dilute bath and as shown in Sec. V, the probe-bath measurement evidently matches dilute shear theory quite well. Nonetheless, one must carefully 
consider contributions to stress due to collisions between the bath particles themselves. The contribution to stress due to bath/bath particle interactions was studied via Brownian dynamics simulation and found not to deviate from the equilibrium osmotic pressure. Thus, we conclude that forcing the probe through the suspension and computing the stress on the probe phase gives an accurate account of nonequilibrium stress in the absence of hydrodynamic interactions, at least for equal probe and bath-particle sizes.

Previous macroscale studies of complex fluids reveal yield-stress behavior in dense colloidal dispersions [LeGrand and Petekidis (2008); Petekidis et al. (2004)] and colloidal glasses and gels [Buscall and White (1987); Potanin and Russel (1996); Saltzman and Schweizer (2006); Gopalakrishnan et al. (2006)], for example. In the case of colloidal glasses, a shear-induced activated hopping may be one mechanism that allows relaxation of the structure, giving rise to an effective yield stress. It would be interesting to consider the single-particle forcing of active microrheology in such a context. In addition, certain weak colloidal gels can sustain their weight temporarily under gravity, but over time undergo slow compaction and at longer times the network may undergo a sudden and dramatic collapse [Poon et al. (1999); Manley et al. (2005)]. It has been proposed that as the network undergoes thermal rearrangements, the effective yield stress of the network evolves, and when this yield stress can no longer balance gravitational stresses, collapse occurs [Manley et al. (2005)]. Similar rupture has been studied in colloidal gels undergoing shear [Sprakel et al. (2011); Lindström et al. (2012)]. An extension of the theory presented here to include attractive potentials between the particles warrants future investigation.

In the results presented here, we have assumed that the probes and bath particles are equally sized, but this need not be the case. The analytical expressions given for the microdiffusivity, the microviscosity, and the stress are for arbitrary size ratio $a / b$, and each is proportional to the scale factor $(1+a / b)$ for all $P e$ when neglecting hydrodynamic interactions. In the limit of a small probe, $a / b \rightarrow 0$, this factor reduces to unity and for $P e \gg 1$ each scales with $P e$ and $\phi_{b}$ as expected. For $a / b \gg 1$, the large-probe limit $(1+a / b) \phi_{b} P e \sim(a / b) \phi_{b} P e$ and remains small [Zia and Brady (2010)]. The size ratio can thus be incorporated into experimental measurement if the sizes of the particles and probes are known. On the other hand, what if the medium itself is of unknown composition? First, because the stress, diffusivity, and viscosity all have the same ratio of probe size to the characteristic length scale of the medium, whether $a / b=1, a / b>1$, or $a / b<1$ does not matter. This ratio is not needed. Even in the presence of hydrodynamic interactions, the equivalence in the scaling of size ratio for stress, diffusion, and viscosity is still present so it still does not matter. But care must be taken when the probe is very large or very small compared to the characteristic length scale of the embedding medium. Here, the only knowledge about the material required in an experiment is an order-of-magnitude estimate of the average length scale in the material, so as to avoid the use of a comparatively very small or large probe. A small probe can be used, but its encounters with the relevant microstructural features may be so infrequent as to require many realizations in order to obtain a statistically relevant number of encounters. One must be careful in this case, because an insufficient amount of data my lead to an incorrect conclusion, e.g., that the mean-square displacement grows nonlinearly in time as one would find in the ballistic regime. At the opposite extreme of a very large probe, care must be taken to be sure the probe is still Brownian in size. While one might consider an approach of using a very large probe to try to replicate macrorheology, the analysis presented here is based on a statistical averaging that allows the probe to diffuse. But in the limit of $a / b \rightarrow \infty$, the probes are not Brownian and detection of fluctuations will be difficult. The force applied to a large 
probe must be very strong in order to drive the suspension from equilibrium, since $D_{a} \rightarrow 0$ for a large probe and the Péclet number of the bath particles $P e=U a / D_{b}$. Hence, the proper size ratio is $a / b \sim O(1)$.

The effect of hydrodynamic interactions between particles was not considered in this study. These are expected to produce a qualitative effect at high $P e$, as lubrication interactions between particles induce shear thickening in the suspension. We expect the same stress-migration relation to hold, and the same scaling of the stress in Pe. Only in the "pure hydrodynamic limit" of no interparticle forces and no Brownian motion should the scaling change, where the $P e$-dependence of the contact microstructure is now less than unity [Khair and Brady (2006)]. When hydrodynamics become important, additional changes in the scaling of probe- to bath-particle size ratio will also become important, as these may strongly affect the microdiffusivity [Davis and Hill (1992); Zia and Brady (2010)].

Since the relation $\partial \Sigma / \partial n \sim R D$ was shown to hold for strongly sheared dilute suspensions [Brady and Morris (1997)], and we have found here good agreement between the $\Sigma^{\text {micro }}$ and $\Sigma^{\text {macro }}$, we believe that the proposed relation (28) also holds for simply sheared suspensions. The shear stresses in this case are fully defined by the viscous term and so we expect that the remaining diffusive terms are equivalent.

In conclusion, we find a new relationship between stress gradients and particle migration in colloidal dispersions away from equilibrium. This relationship accurately predicts normal stresses, osmotic pressure, and normal stress differences in nonlinear microrheology when compared to benchmark theory and to dynamic simulation results. The results are found to be consistent with those found in traditional shear macrorheology and can be applied to dilute and to concentrated dispersions. Importantly, the analytical relationship derived allows two simple measurements, mean and mean-square motion, to reveal the full rheology of a complex fluid: Viscosity, diffusivity, and stress - a significant amount of information gleaned just from watching a particle move. We propose that the expression for the stress thus obtained, $\boldsymbol{D}=-\left(\Sigma^{\text {neq }} / n_{a}+\boldsymbol{P}\right) \cdot \boldsymbol{M}^{\text {neq }}$, can be viewed as a nonequilibrium form of the Stokes-Einstein relation, in which stress is the ratio of fluctuation and dissipation. The stress can be viewed as energy density that is at least partially stored as free energy density in the deformed configuration of bath particles, and the relation $\Sigma \sim n_{a} C T^{\mathrm{eff}}$, as a "nonequilibrium equation of state".

\section{ACKNOWLEDGMENTS}

This work was supported in part by the National Defense Science and Engineering Graduate Fellowship program and by the National Science Foundation Grant No. CBET0931418. The authors thank Dr. James W. Swan for Brownian dynamics code for microrheology.

\section{APPENDIX A: MICRORHEOLOGY MODEL SYSTEM AND BACKGROUND}

The microrheology model system comprises a dispersion of neutrally buoyant colloidal particles of size $b$ immersed in a Newtonian solvent of viscosity $\eta$ and density $\rho$. A set of Brownian probe particles of size $a$ is driven by an external force through the suspension; because the particles are small, $R e \ll 1$ and inertia can be neglected. The number density of probes $n_{a}$ relative to the number density of bath particles $n_{b}$ is small: $n_{a} / n_{b} \ll 1$. As a probe moves through the bath, it drives the suspension from equilibrium. Simultaneously, the Brownian motion of the bath particles acts to recover their equilibrium configuration, giving rise to an entropic restoring force, $k T / b$, where $k T$ is the 
thermal energy of the bath. The degree to which the suspension is driven from equilibrium, and hence its effect on probe motion, is determined by the strength of external probe forcing $\boldsymbol{F}^{\text {ext }}$ compared to thermal restoring force $k T / b$, defining a Péclet number: $P e=F^{\mathrm{ext}} /(k T / b)$. This interplay between probe motion and microstructural response gives rise to changes in probe velocity, which can be used to interrogate suspension properties [Squires and Brady (2005); Meyer et al. (2005); Khair and Brady (2006); Wilson et al. (2009); Zia and Brady (2010)].

In general, the colloidal particles interact through hydrodynamic and interparticle forces which may be both short- and long-ranged; the simplest model for the interactive force, which shall be adopted here, is the hard-sphere potential. Thusly defined, the colloids exert no force on each other until their surfaces touch, i.e., when their separation $r=a+b$. At contact, an infinite potential $V(r)$ prevents their overlap:

$$
V(r)= \begin{cases}\infty, & r<a+b \\ 0, & r>a+b\end{cases}
$$

The radii $a$ and $b$ at which the particles exert the hard-sphere force may or may not be the same as their physical or hydrodynamic radii, $a_{h}$ and $b_{h}$, where the no-slip boundary condition is met. Various physical conditions of the colloid or solvent can extend the effective size of the particle beyond the hydrodynamic radius, e.g., steric repulsion or an ionic screening layer. The particles then repel each other at their extended, or "thermodynamic" radii, $a_{h}$ and $b_{h}$ (Fig. 14). This approach forms the foundation of the excluded annulus model of Morris and Brady (1996), in which the ratio of the two radii ( $\lambda_{a}=a / a_{h}$ and $\lambda_{b}=b / b_{h}$ ) can be modulated to account for the relative importance of hydrodynamic-to-interparticle forces. When $\lambda_{a}, \lambda_{b} \gtrsim O(1)$, the particles are able to approach each other closely enough to experience (long-range) hydrodynamic interactions. For $\lambda_{a}, \lambda_{b} \rightarrow 1$, lubrication interactions also become important. At the opposite extreme of $\lambda_{a}, \lambda_{b} \gg 1$, strong interparticle repulsion keeps the particles sufficiently separated that hydrodynamic interactions are negligibly weak. Although for this study attention is focused on the limit of no hydrodynamic interactions, $\lambda_{a}, \lambda_{b} \rightarrow \infty$, the model captures the essential features of dispersive and dissipative processes while allowing clarity in the exposition of the ideas.

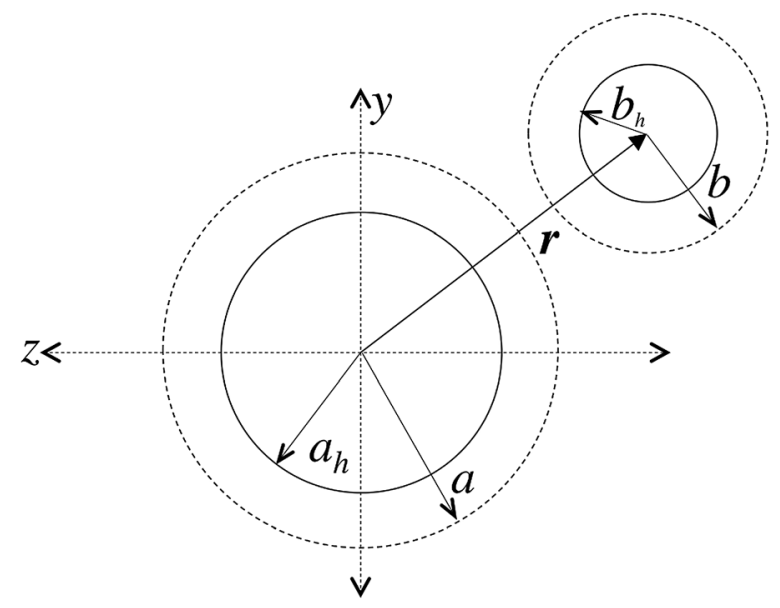

FIG. 14. Hydrodynamic and thermodynamic radii. 


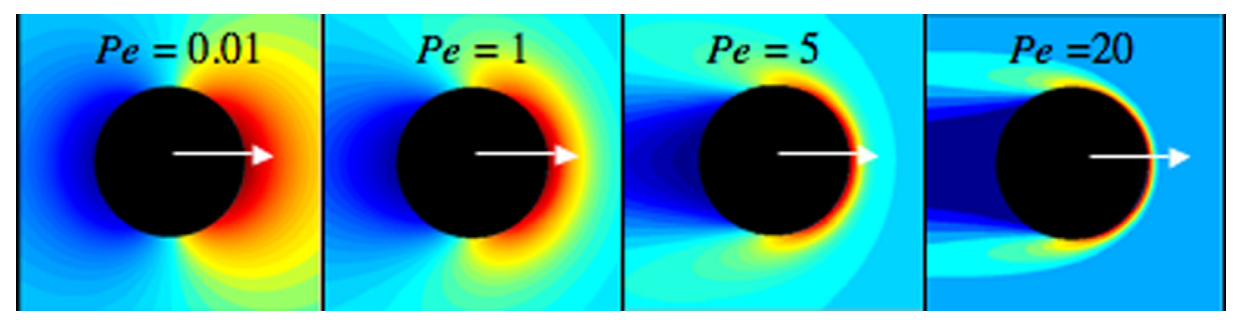

FIG. 15. Theoretical predictions for the deformed microstructure around a moving probe particle in the absence of hydrodynamic interactions at the pair level. The test particle is moving to the right and there is a build-up of background-particle density in front (red) of the probe and a deficit (dark blue) in the trailing wake [Squires and Brady (2005)].

As the probe particle moves through the suspension it must push neighboring particles out of its way; a buildup of background-particle concentration forms in front of the advancing probe and a deficit or wake trails it. Brownian diffusion of the bath particles acts to restore isotropy, but as $P e$ is increased advection wins the competition and the microstructural deformation becomes highly anisotropic. Figure 15 gives an illustration of the deformed microstructure at several values of the Péclet number, where $P e$ increases from left to right in the frames, and a corresponding evolution of microstructural shape can be seen. The microstructure hinders the probe, slowing it down. Squires and Brady (2005) and Khair and Brady (2006) interpreted the mean speed reduction as the viscous drag of the bath, and defined an effective viscosity via application of Stokes' drag law to the average velocity of the probe,

$$
\boldsymbol{F}^{\mathrm{ext}}=6 \pi \eta a \frac{\eta^{\mathrm{eff}}}{\eta}\langle\boldsymbol{U}\rangle .
$$

The effective viscosity is then given by

$$
\frac{\eta^{\mathrm{eff}}}{\eta}=\frac{F^{0}}{6 \pi \eta a\langle U\rangle},
$$

where $\langle U\rangle=-\langle\boldsymbol{U}\rangle \cdot \boldsymbol{F}^{\mathrm{ext}} / F^{\mathrm{ext}}$. The effective viscosity may then be written as

$$
\frac{\eta^{\mathrm{eff}}}{\eta} \approx 1+\frac{\eta^{\mathrm{micro}}}{\eta},
$$

where the microviscosity $\eta^{\text {micro }} / \eta$ is the viscous drag of the particle microstructureabove and beyond the solvent viscosity. In general, it includes contributions due to hydrodynamic, interparticle, and Brownian drag: $\eta^{\text {micro }}=\eta^{\text {micro, } H}+\eta^{\text {micro, } P}+\eta^{\text {micro, } B}$. In the present case, only the interparticle contribution is present, and in the dilute limit, it is an $O\left(\phi_{b}\right)$ effect:

$$
\frac{\eta^{\mathrm{micro}, P}}{\eta}=\frac{3}{4 \pi} P e^{-1} \phi_{b}\left(1+\frac{a}{b}\right)^{2} \boldsymbol{u} \cdot \int \boldsymbol{n} g(1) d \Omega
$$

The same collisions between probe and bath particles that reduce mean probe speed also cause fluctuations in probe velocity. Zia and Brady (2010) found that collisions between probe and bath particles cause the probe to undergo a random-walk process. They show that the long-time mean-square fluctuational motion of the probe is diffusive and determined the 
effective diffusivity of the forced probe for the full range of Péclet number. They derived the force-induced diffusion, or "microdiffusivity," for a dilute suspension of colloids, in the absence of hydrodynamic interactions. For a probe of size $a$ in a suspension of bath particles of size $b$ at volume fraction $\phi_{b}$, the "microdiffusivity," is given by

$$
\frac{\boldsymbol{D}^{\text {micro }}}{D_{a}}=\frac{3}{4 \pi} \phi_{b}\left(1+\frac{a}{b}\right)^{2} \oint \boldsymbol{n} \boldsymbol{d}^{\prime} d \Omega,
$$

where $D_{a}$ is the bare diffusivity of a probe alone in a solvent and $\boldsymbol{d}^{\prime}$ is the fluctuation vector describing the collision-induced fluctuations in probe motion. At small $\mathrm{Pe}$ Brownian motion dominates and the diffusive behavior of the probe characteristic of passive microrheology is recovered, but with an incremental flow-induced microdiffusivity that scales as $D^{\text {micro }} \sim D_{a} P e^{2} \phi_{b}$, where $\phi_{b}$ is the volume fraction of bath particles and $D_{a}$ is the self-diffusivity of an isolated probe. At the other extreme of high Péclet number, the fluctuational motion is still diffusive, and the diffusivity becomes primarily force-induced, scaling as $D^{\text {micro }} \sim D_{a} P e \phi_{b}$. The force-induced microdiffusivity is anisotropic, with diffusion longitudinal to the direction of forcing larger in both limits compared to transverse diffusion, but more strongly so in the high $P e$ limit.

Finally, the stress is given by [McQuarrie (1976); Brady (1993)]

$$
-\frac{\langle\Sigma\rangle}{n_{a} k T}=1+\frac{3}{8 \pi} \phi_{b}\left(1+\frac{a}{b}\right)^{3} \int_{r=a+b} \boldsymbol{n n} g(\boldsymbol{r}) d \Omega,
$$

where $\boldsymbol{n}$ is the normal to the contact surface at probe-bath particle separation $r=a+b$, and $g(\boldsymbol{r})$ is the probe-bath pair-distribution function at contact. Equation (A6) is an exact formula (for hard spheres) of the stress contribution from the probe particles no matter what the concentration of bath (or probe) particles and for any type of forcing, e.g., shearing motion or an external force for microrheology.

\section{APPENDIX B: CONSTANT EXTERNAL FORCE VERSUS CONSTANT EXTERNAL VELOCITY}

Active microrheology experiments are carried out by two main approaches: driving the probe with a constant external force-e.g., with magnetic tweezers [Habdas et al. (2004)] — or holding the probe fixed in an optical trap and moving the bath past it at a constant velocity-e.g., with laser tweezers [Furst (2005); Meyer et al. (2005)]. In the former case (to which we shall refer as mode 1), the applied probe force remains constant while its velocity fluctuates and, as a result, the probe experiences a collision-induced diffusive spread of its trajectory [Zia and Brady (2010)]. In the latter case (mode 2), the velocity is held constant and cannot fluctuate in magnitude or direction, and hence cannot diffuse (but experiences a fluctuating force instead). As noted above, the theoretical framework for nonlinear microrheology first developed by Squires and Brady (2005) interprets the average probe velocity as the effective viscosity of the suspension, where the average is taken over all possible configurations of the microstructure. They presented the solution for the constant-force microstructure and the corresponding effective viscosity. Although the constant-velocity microstructure was not computed explicitly, they propose that the mode- 2 viscosity should be twice the mode- 1 viscosity,

$$
\frac{\eta_{U}^{\mathrm{micro}}}{\eta}=2 \frac{\eta_{F}^{\mathrm{micro}}}{\eta}
$$


based on a scaling argument justified physically as follows: As the probe moves through the bath, it encounters variations in local bath-particle density. In mode 1, since probe velocity can fluctuate, the probe can "wiggle" around and adjust its speed if it reaches a more dense or less dense region. On the other hand, in mode 2 , the probe velocity cannot change, and it must "bulldoze" through the suspension regardless of fluctuations in local density. This difference is captured by the scaling of the diffusivity. Recall that the Péclet number can be defined as $P e=U(a+b) / D_{r}$, where $D_{r}$ is the appropriate diffusive scaling. In mode 1 , the proper diffusive scaling is the relative diffusivity $D_{F}$ between a pair of particles:

$$
D_{F} \sim D_{a}+D_{b}=\frac{k T}{6 \pi \eta}\left(\frac{1}{a}+\frac{1}{b}\right) \quad(\text { constant force })
$$

where $D_{a}=k T / 6 \pi \eta a$ and $D_{b}=k T / 6 \pi \eta b$ are the isolated diffusivities of a probe and a bath particle, respectively. But in mode 2 , since the probe cannot diffuse, the proper diffusive scaling $D_{U}$ is that of the bath particles, which can diffuse:

$$
D_{U} \sim D_{b}=\frac{k T}{6 \pi \eta b} \quad(\text { constant velocity })
$$

For a probe-to-bath-particle size ratio $a / b=1$, we then have that $D_{F}=2 D_{U}$, with the inverse ratio for the effective viscosity. These scaling arguments apply in the limit of no hydrodynamic interactions. Carpen and Brady (2005) evaluated this prediction via Brownian-dynamics simulation of steady-state microrheology and found agreement between simulation and theory.

\section{References}

Barnes, H. A., F. F. Hutton, and K. Walters, An Introduction to Rheology (Elsevier, Oxford, UK, 1989).

Batchelor, G. K., "Sedimentation in a dilute dispersion of spheres," J. Fluid Mech. 52(2), 245-268 (1972).

Batchelor, G. K., "Brownian diffusion of particles with hydrodynamic interaction," J. Fluid Mech. 74, 1-29 (1976).

Batchelor, G. K., "The effect of Brownian motion on the bulk stress in a suspension of spherical particles," J. Fluid Mech. 83, 97-117 (1977).

Bausch, A. R., F. Ziemann, A. A. Boulbitch, K. Jacobson, and E. Sackmann, "Local measurements of viscoelastic parameters of adherent cell surfaces by magnetic bead rheometry,” Biophys. J. 75, 2038-2049 (1998).

Bergenholtz, J., J. F. Brady, and M. Vicic, "The non-Newtonian rheology of dilute colloidal suspensions," J. Fluid Mech. 456, 239-275 (2002).

Brady, J. F., "Brownian motion, hydrodynamics, and the osmotic pressure," J. Chem. Phys. 98, 3335-3341 (1993).

Brady, J. F., and J. F. Morris, "Microstructure of strongly sheared suspensions and its impact on rheology and diffusion,” J. Fluid. Mech. 348, 103-139 (1997).

Brady, J. F., and M. Vicic, "Normal stresses in colloidal suspensions," J. Rheol. 39(3), 545-566 (1995).

Breedveld, V., and D. J. Pine, "Microrheology as a tool for high-throughput screening," J. Mater. Sci. 38, 4461-4470 (2003).

Buscall, R., and L. R. White, "The consolidation of concentrated suspensions.Part 1. The theory of sedimentation,” J. Chem. Soc., Faraday Trans. 1 83, 873-891 (1987).

Carnahan, N. F., and K. E. Starling, "Equation of state for nonattracting rigid spheres," J. Chem. Phys. 51(2), 635-636 (1969). 
Carpen, I. C., and J. F. Brady, "Microrheology of colloidal dispersions by Brownian dynamics simulations," J. Rheol. 49, 1483-1502 (2005).

Crocker, J. C., "Measurement of the hydrodynamic corrections to the Brownian motion of two colloidal spheres," J. Chem. Phys. 106, 2837-2840 (1997).

Crocker, J. C., and D. G. Grier, "Methods of digital video microscopy for colloidal studies," J. Colloid Interface Sci. 179, 298-310 (1996).

Crocker, J. C., J. A. Matteo, A. D. Dinsmore, and A. G. Yodh, "Entropic attraction and repulsion in binary colloids probed with a line optical tweezer," Phys. Rev. Lett. 82, 4352-4355 (1999).

Crocker, J. C., M. T. Valentine, E. R. Weeks, T. Gisler, P. D. Kaplan, A. G. Yodh, and D. A. Weitz, “Two-point microrheology of inhomogeneous soft materials," Phys. Rev. Lett. 85(4), 888-891 (2000).

Davis, R. H., and N. A. Hill, "Hydrodynamic diffusion of a sphere sedimenting through a dilute suspension of neutrally buoyant spheres," J. Fluid Mech. 236, 513-533 (1992).

DeGroot, S. R., and P. Mazur, Non-Equilibrium Thermodynamics (Dover, General Publishing Co., Ltd., Ontario, Canada, 1984).

Einstein, A., "Investigations on the theory of the Brownian movement," Ann. Phys. 19, 371-381 (1906).

Foss, D. R., and J. F. Brady, "Brownian dynamics simulation of hard-sphere colloidal dispersions," J. Rheol. 44(3), 629-651 (2000a).

Foss, D. R., and J. F. Brady, "Structure, diffusion and rheology of Brownian suspensions by Stokesian dynamics simulation,” J. Fluid Mech. 407, 167-200 (2000b).

Freundlich, H., and W. Seifriz, "Über die elästizitaet von solen und gelen," Z. Phys. Chem. 104, 233-261 (1923).

Furst, E. M., “Applications of laser tweezers in complex fluid rheology,” Curr. Opin. Colloid Interface Sci. 10, 79-86 (2005).

Gisler, T., and D. A. Weitz, "Scaling of the microrheology of semidilute f-actin solutions," Phys. Rev. Lett. 82, 1606-1610 (1999).

Gopalakrishnan, V., K. S. Schweizer, and C. F. Zukoski, "Linking single particle rearrangements to delayed collapse times in transient depletion gels," J. Phys.: Condens. Matter 18, 11531-11550 (2006).

Guilford, W. H., R. C. Lantz, and R. W. Gore, "Locomotive forces produced by single leukocytes in vivo and in vitro,” Am. J. Physio-Cell. Ph. 268(5), C1308-C1312 (1995).

Habdas, P., D. Schaar, A. C. Levitt, and E. R. Weeks, "Forced motion of a probe particle near the colloidal glass transition,” Europhys. Lett. 67, 477-483 (2004).

Heath, J. R., M. E. Davis, and L. Hood, “Nanomedicine-revolutionizing the fight against cancer,” Sci. Am. 300, 44-51 (2009).

Heyes, D. M., and J. R. Melrose, "Brownian dynamics simulations of model hard-sphere suspensions," J. NonNewtonian Fluid Mech. 46, 1-28 (1993).

Khair, A. S., and J. F. Brady, "Microviscoelasticity of colloidal dispersions," J. Rheol. 49, 1449-1481 (2005).

Khair, A. S., and J. F. Brady, "Single particle motion in colloidal dispersions: A simple model for active and nonlinear microrheology," J. Fluid Mech. 557, 73-117 (2006).

Khair, A. S., and J. F. Brady, "Microrheology of colloidal dispersions: Shape matters," J. Rheol. 52, 165-196 (2008).

Lau, A. W. C., B. D. Hoffman, A. Davies, J. C. Crocker, and T. C. Lubensky, "Microrheology, stress fluctuations, and active behavior of living cells," Phys. Rev. Lett. 91, 198101 (2003).

LeGrand, A., and G. Petekidis, "Effects of particle softness on the rheology and yielding of colloidal glasses," Rheol. Acta 47(5-6), 579-590 (2008).

Levine, A. J., and T. C. Lubensky, “One- and two-particle microrheology,” Phys. Rev. Lett. 85, 1774-1778 (2000).

Lindström, S. B., T. E. Kodger, J. Sprakel, and D. A. Weitz, "Structures, stresses, and fluctuations in the delayed failure of colloidal gels," Soft Matter 8, 3657-3664 (2012).

Lu, Q., and M. J. Solomon, "Probe size effects on the microrheology of associating polymer solutions," Phys. Rev. E 66, 061504 (2002).

Lukacs, G. L., P. Haggie, O. Seksek, D. Lechardeur, N. Freedman, and A. S. Verkman, "Size-dependent DNA mobility in cytoplasm and nucleus,” J. Bio. Chem. 275(3), 1625-1629 (1999). 
MacKintosh, F. C., and C. F. Schmidt, "Microrheology,” Curr. Opin. Colloid Interface Sci. 4, 300-307 (1999).

Manley, S., J. M. Skotheim, L. Mahadevan, and D. A. Weitz, "Gravitational collapse of colloidal gels," Phys. Rev. Lett. 94, 218302 (2005).

Mason, T. G., and D. A. Weitz, "Optical measurements of frequency-dependent linear viscoelastic moduli of complex fluids," Phys. Rev. Lett. 74(7), 1250-1253 (1995).

Mason, T. G., K. Ganesan, J. H. vanZanten, D. Wirtz, and S. C. Kuo, "Particle tracking microrheology of complex fluids," Phys. Rev. Lett. 79(17), 3282-3286 (1997).

McQuarrie, D. A., Statistical Mechanics (Harper and Row, New York, 1976).

Meyer, A., A. Marshall, B. G. Bush, and E. M. Furst, "Laser tweezer microrheology of a colloidal suspension," J. Rheol. 50, 77-92 (2005).

Morris, J. F., and J. F. Brady, "Self diffusion in sheared suspensions," J. Fluid. Mech. 312, 223-252 (1996).

Olsen, B. D., J. A. Kornfield, and D. A. Tirrell, "Yielding behavior in injectable hydrogels from telechelic proteins," Macromolecules 43(21), 9094-9099 (2010).

Perrin, J-.B., "Mouvement Brownian et réalité moléculaire (Brownian motion and the molecular reality)," Ann. Chim. Phys. 18, 5-114 (1909).

Petekidis, G., D. Vlassopoulous, and P. N. Pusey, "Yielding and flow of sheared colloidal glasses," J. Phys.: Condens. Matter 16(8), S3955-S3963 (2004).

Poon, W. C. K., L. Starrs, S. P. Meeker, A. Moussad, R. M. L. Evans, P. N. Pusey, and M. M. Robins, "Delayed sedimentation of transient gels in colloid-polymer mixtures: Dark-field observation, rheology and dynamic light scattering studies," Faraday Discuss. 112, 143-154 (1999).

Potanin, A. A., and W. B. Russel, "Fractal model of consolidation of weakly aggregated colloidal dispersions," Phys. Rev. E 53, 3702-3709 (1996).

Rallison, J. M., and E. J. Hinch, "The effect of particle interactions on dynamic light scattering from a dilute suspension,” J. Fluid Mech. 167, 131-168 (1986).

Saltzman, E. J., and K. S. Schweizer, "Activated hopping and dynamical fluctuation effects in hard sphere suspensions and fluids," J. Chem. Phys. 125(4), 044509-1-044509-19 (2006).

Sami, S., "Stokesian dynamics simulation of Brownian suspensions in extensional flow," Ph.D. thesis, California Institute of Technology, 1996.

Schultz, K. M., and E. M. Furst, "High-throughput rheology in a microfluidic device," Lab Chip 11, 3802-3809 (2011).

Sprakel, J., S. B. Lindström, T. E. Kodger, and D. A. Weitz, "Stress enhancement in the delayed yielding of colloidal gels," Phys. Rev. Lett. 106, 248303 (2011).

Squires, T. M., "Nonlinear microrheology: Bulk stresses versus direct interactions," Langmuir 24, 1147-1159 (2008).

Squires, T. M., and J. F. Brady, “A simple paradigm for active and nonlinear microrheology,” Phys. Fluids 17, 073101 (2005).

Sriram, I., R. DePuit, T. M. Squires, and E. M. Furst, "Small amplitude active oscillatory microrheology of a colloidal suspension,” J. Rheol. 53, 357-381 (2009).

Suh, J., D. Wirtz, and J. Hanes, "Efficient active transport of gene nanocarriers to the cell nucleus," Proc. Natl. Acad. Sci. U.S.A. 100(7), 3738-3882 (2003).

Verkman, A. S., "Solute and macromolecule diffusion in cellular aqueous compartments," Trends Biochem. Sci. 27, 27-33 (2002).

Wilson, L. G., A. W. Harrison, A. B. Schofield, J. Arlt, and W. C. K. Poon, "Passive and active microrheology of hard-sphere colloids," J. Phys. Chem. 113, 3806-3812 (2009).

Zia, R. N., "Individual particle motion in colloids: Microviscosity, microdiffusivity, and normal stresses," Ph.D. thesis, California Institute of Technology, 2011.

Zia, R. N., and J. F. Brady, "Single particle motion in colloids: Force-induced diffusion," J. Fluid Mech. 658, 188-210 (2010).

Zia, R. N., and J. F. Brady, "Stress development, relaxation, and memory in colloidal dispersions: Transient nonlinear microrheology," J. Rheol. (submitted).

Ziemann, F., J. Radler, and E. Sackmann, "Local measurements of viscoelastic moduli of entangled actin networks using an oscillating magnetic bead micro-rheometer,” Biophys. J. 66, 2210-2216 (1994). 